\title{
CONFORMAL SYMMETRY, UNITARITY, AND THE EQUATIONS OF MOTION FOR STRINGS IN BACKGROUND FIELDS
}

\author{
Ratindranath AKHOURY and Yasuhiro OKADA \\ Randall Laboratory of Physics, University of Michigan, Ann Arbor, Michigan 48109, USA
}

Received 28 July 1987

\begin{abstract}
We impose the constraint of the unitarity of the $S$-matrix on the theory of a closed bosonic string propagating in a background of some of its modes. The equations of motion for not only the massless but also for some of the massive modes of the string are thus consistently obtained in a weak field expansion. To a given order in the weak field expansion, they contain contributions to all orders in $\alpha^{\prime}$. We also show that conformal invariance guarantees that the $S$-matrix be unitary.
\end{abstract}

\section{Introduction}

Conformal invariance plays a fundamental role in the formulation of a consistent string theory. For strings in flat space-time it is necessary to show the decoupling of the unphysical degrees of freedom in a covariant approach. In the case of strings in background fields, conformal invariance has been shown [1] to provide the important connection between a two-dimensional field theory and string dynamics in $D$-dimensional space-time. This connection is realized by requiring the $\beta$-functions of the non-linear sigma model to vanish, thereby giving rise to the classical equations of motion for the background. In most of these approaches an expansion in the $\alpha^{\prime}$, the inverse string tension is utilized. It would be interesting to see what constraints the background fields must satisfy by imposing directly the requirement of the unitarity of the $S$-matrix and to investigate the interrelations between conformal symmetry and unitarity. It is precisely these issues that this paper addresses.

We investigate, in this paper, the theory of a closed bosonic string propagating in a background consisting of a condensate of some of its modes (massless, tachyon and other massive mode). We formulate a unitarity condition, which is essentially the requirement that physical "in" states map into physical "out" states after interactions. When this condition is imposed on the theory, we find that the background fields must satisfy certain constraints which are just the equations of motion for these modes. For explicit calculations, we do not use the Polyakov path-integral formulation of the string theory and neither do we make an expansion 
in $\alpha^{\prime}$. Rather, we use the string operator formalism and make a weak field expansion around flat space-time. The result is that the equations of motion, not only for the massless modes of the string but also for the tachyon and other massive mode, can be consistently obtained to a given order in a weak field expansion. (In this paper we present results up to the second order in weak fields.) These equations of motion for the various modes, to a given order in the weak field, contain contributions to all orders in $\alpha^{\prime}$, are consistent with the string tree-amplitude calculations, and are compatible with each other. We also show how the unitarity condition is related to conformal invariance. Specifically, we show to an arbitrary order in the weak field expansion that the closure of the Virasoro algebra for strings in background fields guarantees that the $S$-matrix is unitary. A summary of the results arrived here has been published in ref. [2].

The paper is organized as follows: In sect. 2 we present the basic formalism used in this paper. In subsect. 2.1 we discuss the first quantization of the closed bosonic string in the presence of background fields, and introduce the weak field expansion. In subsect. 2.2 we formulate the unitarity condition as the requirement that a certain matrix element should vanish. Subsect. 2.3 contains a discussion of the relation of this constraint with the closure of the Virasoro algebra. In sect. 3 we present the explicit calculations of the equations of motion in a weak field expansion, using the unitarity constraint. Subsect. 3.1 contains the result for the free equations of motion for the massless, tachyon and the first massive modes. In subsect. 3.2 we go to the next order in the weak field expansion and obtain the interaction terms in these equations of motion. Sect. 4 contains a discussion of our results and technical details of sects. 2 and 3 are included in appendices A, B and C.

\section{Formalism}

\subsection{WEAK FIELD EXPANSION}

In this section, we will consider the first quantization of a closed bosonic string theory propagating in the presence of space-time background fields. Our approach is based on hamiltonian formulation and we will use a weak field expansion around a flat space-time. The basic formalism we follow in this paper is explained in detail in our previous paper [3], so here we will just review the procedure briefly.

Let us start with the following action

$$
S=-\frac{1}{4 \pi \alpha^{\prime}} \int \mathrm{d} \sigma \mathrm{d} \tau\left\{\sqrt{-\gamma} \gamma^{\alpha \beta} g_{\mu \nu}(X) \partial_{\alpha} X^{\mu} \partial_{\beta} X^{\nu}+\varepsilon^{\alpha \beta} B_{\mu \nu}(X) \partial_{\alpha} X^{\mu} \partial_{\beta} X^{\nu}\right\},
$$

where $g_{\mu \nu}(X)$ and $B_{\mu \nu}(X)$ represent condensates of background massless modes of the string theory, i.e., a symmetric tensor field for $g_{\mu \nu}(X)$ and an antisymmetric tensor field for $B_{\mu \nu}(X)$. In our approach the dilaton mode is included in a trace part 
of $g_{\mu \nu}(X) . \gamma_{\alpha \beta}(\sigma \tau)$ is the two-dimensional metric tensor and $\varepsilon^{\alpha \beta}$ is a two-dimensional antisymmetric tensor which satisfies $\varepsilon^{01}=1$. From now on, we only consider the theory at the critical dimension, i.e., $D=26$. In ref. [3] starting from the above action, we formulated the classical Hamilton system first and quantized it by replacing Poisson brackets by canonical commutators. When we pass from the classical to the quantum theory, we encounter the operator ordering problems. In ref. [3], we introduced a weak field expansion around flat space-time $\eta_{\mu \nu}$, namely

$$
\begin{aligned}
& g_{\mu \nu}(X)=\eta_{\mu \nu}+h_{\mu \nu}(X) \\
& B_{\mu \nu}(X)=b_{\mu \nu}(X) .
\end{aligned}
$$

We regard $h_{\mu \nu}(X)$ and $b_{\mu \nu}(X)$ as weak fields and expand the hamiltonian and the Virasoro operators with respect to these weak fields. The zeroth order hamiltonian is the free hamiltonian in the conformal gauge because it is in fact the hamiltonian in the flat case. Then we can treat the $h_{\mu \nu}(X), b_{\mu \nu}(X)$ parts as perturbations and define the normal ordering of operators with respect to this free hamiltonian. Going to the interaction representation, the Virasoro operators and the hamiltonian are expressed in terms of operators $\pi_{\mu}^{\text {in }}(\tau \sigma)$ and $X^{\mu(i n)}(\tau \sigma)$ which satisfy the free equations of motion

$$
\begin{aligned}
X^{\mu(\mathrm{in})} & =x^{\mu}+\frac{1}{2}\left(2 \alpha^{\prime}\right) p_{\mu} \tau+\frac{1}{2} \sqrt{2 \alpha^{\prime}} \sum_{n \neq 0} \frac{1}{n}\left(\alpha_{n}^{\mu} \mathrm{e}^{-i n(\tau-\sigma)}+\tilde{\alpha}_{n}^{\mu} \mathrm{e}^{-i n(\tau+\sigma)}\right. \\
\pi_{\mu}^{(\text {in })} & =\frac{1}{2 \alpha^{\prime} \pi} \eta_{\mu \nu} \frac{\partial X^{\nu(\text { in })}}{\partial \tau}
\end{aligned}
$$

Here, $p^{\mu}, x^{\mu}, \alpha_{n}^{\mu}$ satisfy the following commutation relations

$$
\begin{aligned}
& {\left[x^{\mu}, p^{\nu}\right]=i \eta^{\mu \nu},} \\
& {\left[\alpha_{n}^{\mu}, \alpha_{m}^{\nu}\right]=n \delta_{n+m, 0} \eta^{\mu \nu},} \\
& {\left[\tilde{\alpha}_{n}^{\mu}, \tilde{\alpha}_{m}^{\nu}\right]=n \delta_{n+m, 0} \eta^{\mu \nu} .}
\end{aligned}
$$

Expanding $L_{n}$ and $\bar{L}_{n}$ according to the number of weak fields

$$
\begin{aligned}
& L_{n}=L_{n}^{(0)}+L_{n}^{(1)}+L_{n}^{(2)}+\ldots \\
& \bar{L}_{n}=\bar{L}_{n}^{(0)}+\bar{L}_{n}^{(1)}+\bar{L}_{n}^{(2)}+\ldots
\end{aligned}
$$

we obtain expressions for the Virasoro operators. (Hereforth we will omit the 
superscript "in" and use units in which $\alpha^{\prime}=\frac{1}{2}$ ).

$$
\begin{aligned}
& L_{n}^{(0)}=\frac{1}{4 \pi} \int_{0}^{2 \pi} \mathrm{d} \sigma \mathrm{e}^{-i n \sigma}: P^{\mu}(\tau \sigma)^{2}, \\
& \bar{L}_{n}^{(0)}=\frac{1}{4 \pi} \int_{0}^{2 \pi} \mathrm{d} \sigma \mathrm{e}^{i n \sigma}: \tilde{P}_{\mu}(\tau \sigma)^{2} ; \\
& L_{n}^{(1)}=L_{-n}^{(1)}=\frac{1}{4 \pi} \int_{0}^{2 \pi} \mathrm{d} \sigma \mathrm{e}^{-i n \sigma}: \rho_{\mu \nu}(X) p^{\mu}(\tau \sigma) \tilde{p}^{\nu}(\tau \sigma), \\
& L_{n}^{(2)}=\bar{L}_{-n}^{(2)}=\frac{1}{4 \pi} \int_{0}^{2 \pi} \mathrm{d} \sigma \mathrm{e}^{-i n \sigma} J^{\mu 2}
\end{aligned}
$$

and for the hamiltonian

$$
H=H^{(0)}+H^{(1)}+H^{(2)}+\ldots,
$$

where the $H^{(i)}$ 's are given by

$$
\begin{aligned}
& H^{(0)}=L_{0}^{(0)}+\bar{L}_{0}^{(0)}-2, \\
& H^{(1)}=2 L_{0}^{(1)}, \\
& H^{(2)}=2 L_{0}^{(2)}, \text { etc. }
\end{aligned}
$$

Here, we have used the following

$$
\begin{aligned}
P^{\mu}(\tau \sigma) & =\left(\frac{\partial}{\partial \tau}-\frac{\partial}{\partial \sigma}\right) X^{\mu}(\tau \sigma) \\
& =\frac{1}{2} p^{\mu}+\sum_{n \neq 0} \alpha_{n}^{\mu} \mathrm{e}^{-i n(\tau-\sigma)} \\
\tilde{P}^{\nu}(\tau \sigma) & =\left(\frac{\partial}{\partial \tau}+\frac{\partial}{\partial \sigma}\right) X^{\nu}(\tau \sigma) \\
& =\frac{1}{2} p^{\nu}+\sum_{n \neq 0} \tilde{\alpha}_{n}^{\nu} \mathrm{e}^{-i n(\tau+\sigma)}, \\
\rho_{\mu \nu}(X) & \equiv-h_{\mu \nu}(X)+b_{\mu \nu}(X), \\
J_{\mu}(X) & \equiv \frac{1}{2}\left(p^{\lambda} \rho_{\lambda \mu}(X)+\rho_{\mu \lambda}(X) \tilde{p}^{\lambda}\right),
\end{aligned}
$$


and all the contraction of space-time indices is taken by using the flat metric $\eta_{\mu \nu}$ and $\eta^{\mu \nu}$.

In the above expressions, we have introduced the normal ordering for the oscillator parts as is normally done in the flat-space case. However, in the expression like $L_{n}^{(1)}$, we still have an ordering ambiguity for the zero mode part ( $p^{\mu}$ and $x^{\nu}$ ). From now on, we assume the following constraints on $\rho_{\mu \nu}(X)$ in order to remove this ambiguity.

$$
\partial^{\mu} \rho_{\mu \nu}(X)=\partial^{\nu} \rho_{\mu \nu}(X)=0
$$

Later, we will see that these constraints correspond to the gauge conditions for the background fields. The ordering presumption for $H^{(2)}$ is as yet unspecified. This will be done in subsect. 3.2 and appendix B by requiring that the Nambu-Mathews theorem be valid.

Up to now, we have only considered its background fields of the massless modes. Now we want to take into account the effects of the tachyon and other massive tensor field condensates. To do so, we should add to our action terms which represent the condensates of each modes, or in the hamiltonian formalism using a weak field expression, we can simply add the corresponding terms in the interaction hamiltonian. For the massless case, the form of the interaction hamiltonian which represents the coupling of background fields is, as is seen in eqs. (2.8) and (2.11), determined essentially by the corresponding vertex operator at the first order level. The only difference here is that in the interaction hamiltonian, we don't require the mass-shell condition from the first whereas for the vertex operator we usually assume it for the polarization tensors. In the same way, we can determine the interaction hamiltonian for other modes, for example, for tachyon and for the first massive level, the first order interaction hamiltonian take the following forms

$$
\begin{aligned}
\Delta H_{\mathrm{tach}}^{(1)} & =\frac{1}{2 \pi} \int \mathrm{d} \sigma: \phi(X):, \\
\Delta H_{1 \text { st massive }}^{(1)} & =\frac{1}{2 \pi} \int \mathrm{d} \sigma: M_{\mu \alpha, \nu \beta}(X) P^{\mu} P^{\alpha} \tilde{P}^{\nu} \tilde{P}^{\beta}:,
\end{aligned}
$$

where we assume the following "gauge" conditions

$$
\begin{aligned}
& \partial^{\mu} M_{\mu \alpha, \nu \beta}=\partial^{\alpha} M_{\mu \alpha, \nu \beta}=\partial^{\nu} M_{\mu \alpha, \nu \beta}=\partial^{\beta} M_{\mu \alpha, \nu \beta}=0 \\
& \eta^{\mu \alpha} M_{\mu \alpha, \nu \beta}=\eta^{\nu \beta} M_{\mu \alpha, \nu \beta}=0
\end{aligned}
$$

As we will see later, these operators are sufficient for the order to which we discuss in this paper. For the tachyon modes, one can consider eq. (2.17) only even for higher order terms in the number of tachyon fields. 


\subsection{UNITARITY CONSTRAINT}

In this section, we will discuss what constraints should be satisfied for background fields from the requirement of the unitarity of the $S$ matrix. In general, for the first quantized string theory, conformal symmetry seems to be the necessary condition for consistency. For string theory in a flat background, the no ghost theorem [4] can be proven using the Virasoro algebra with a correct central change term, if we specify the physical states in the usual manner [4]. We would like to address ourself to the corresponding problem in the curved case. Within perturbation theory, we can formulate the condition of unitarity as follows.

First, we assume that the on-shell physical state conditions for the "in" states are the same as those in the flat case, i.e.

$$
\begin{aligned}
& \left.\left.\left(L_{0}^{(0)}\left(\phi_{\text {in }}\right)+\bar{L}_{0}^{(0)}\left(\phi_{\text {in }}\right)-2\right) \mid \text { phys }\right\rangle=0 \quad \text { (on-shell condition }\right), \\
& \left(L_{0}^{(0)}\left(\phi_{\text {in }}\right)-\bar{L}_{0}^{(0)}\left(\phi_{\text {in }}\right) \mid \text { phys }\right\rangle=0, \\
& \left.L_{n}^{(0)}\left(\phi_{\text {in }}\right) \mid \text { phys }\right\rangle=0 \quad(n>0), \\
& \text { (physical state conditions) } \\
& \left.\bar{L}_{n}^{(0)}\left(\phi_{\text {in }}\right) \mid \text { phys }\right\rangle=0 \quad(n>0) .
\end{aligned}
$$

Notice that by using the adiabatic hypothesis, we reduce $L_{n}$ and $\bar{L}_{n}$ to the corresponding flat space expression $L_{n}^{(0)}$ and $\bar{L}_{n}^{(0)}$ at $\tau \rightarrow-\infty$. If the string propagates in the presence of background fields, it can interact at intermediate times with these fields and these effects are represented by the interaction hamiltonian. Therefore, even though we specify the physical states at $\tau=\infty$, we have to make sure that these physical state conditions are preserved after interactions. This requirement can be expressed using the "out" fields, which are the asymptotic fields at $\tau \rightarrow+\infty$, as follows

$$
\begin{aligned}
\left(L_{0}^{(0)}\left(\phi_{\text {out }}\right)+\bar{L}_{0}^{(0)}\left(\phi_{\text {out }}\right)-2\right)|\mathrm{phys}\rangle & =0, \\
\left(L_{0}^{(0)}\left(\phi_{\text {out }}\right)-\bar{L}_{0}^{(0)}\left(\phi_{\text {out }}\right)\right)|\mathrm{phys}\rangle & =0, \\
L_{n}^{(0)}\left(\phi_{\text {out }}\right)|\mathrm{phys}\rangle & =0 \quad(n>0), \\
\bar{L}_{n}^{(0)}\left(\phi_{\text {out }}\right)|\mathrm{phys}\rangle & =0 \quad(n>0) .
\end{aligned}
$$

Using the relation between the "in" and "out" fields

$$
\phi_{\text {out }}=S^{+} \phi_{\text {in }} S \text {, }
$$


the above condition reduces to the following

$$
\begin{aligned}
\left.\left(L_{n}^{(0)}\left(\phi_{\text {in }}\right)+\bar{L}_{0}^{(0)}\left(\phi_{\text {in }}\right)-2\right) S \mid \text { phys }\right\rangle & =0, \\
\left.\left(L_{0}^{(0)}\left(\phi_{\text {in }}\right)-\bar{L}_{0}^{(0)}\left(\phi_{\text {in }}\right)\right) S \mid \text { phys }\right\rangle & =0, \\
\left.L_{0}^{(0)}\left(\phi_{\text {in }}\right) S \mid \text { phys }\right\rangle & =0 \quad(n<0), \\
\left.\bar{L}_{0}^{(0)}\left(\phi_{\text {in }}\right) S \mid \text { phys }\right\rangle & =0 \quad(n<0) .
\end{aligned}
$$

Here $S$ represents the $S$ matrix generated by the interaction hamiltonian

$$
S=T \exp \left(-i \int_{-\infty}^{\infty} H_{\mathrm{I}}\left(\phi_{\text {in }}\right) \mathrm{d} \tau\right)
$$

where the total hamiltonian is decomposed as

$$
H=H^{(0)}+H_{\mathrm{I}}
$$

and $H_{\mathrm{I}}$ is the interaction hamiltonian which contains contributions from all the modes.

In actual calculations, we require the following statement. Take a physical state $|B\rangle$ which satisfies the conditions (2.20) and consider the matrix element

$$
T=\left\langle A\left|L_{n}^{(0)} S\right| B\right\rangle
$$

with $\langle A| L_{n}^{(0)}$ an on-shell, i.e., $\langle A|\left(H^{(0)}+n\right)=0$, but not necessarily a physical state. Then, unitarity demands that $T$ vanish for any choice of states $|A\rangle$ and $|B\rangle$.

As we mentioned earlier, the unitarity of $S$ matrix has close connection with the closure of the Virasoro algebra in the flat case. In the curved case, if we treat the theory classically, we can show the classical Virasoro algebra with Poisson brackets is closed without any constraints on background fields [3]. On the other hand, quantum-mechanically there occur anomalous terms in Virasoro algebra which depend on the background fields and by demanding that these anomalous contributions vanish, we can obtain some constraint equations which correspond to equations of motion for the background fields [3]. In the next section we will show that if the Virasoro algebra is closed, then the above matrix element $T$ vanishes order by order in perturbation theory. Therefore, demanding that $T$ vanish is equivalent to the requirement of the closure of Virasoro algebra, and we can expect some kind of constraint equations for background fields from an explicit computation of the matrix element $T$. This will be done in sect. 3 . 
2.3. EQUIVALENCE BETWEEN THE UNITARITY CONSTRAINT AND THE CLOSURE OF THE VIRASORO ALGEBRA

In this section we will show that the matrix element $T$ vanishes if we assume the closure of the Virasoro algebra. Let us assume that the following Virasoro algebra holds without any anomalous contributions:

$$
\begin{aligned}
& {\left[L_{n}, L_{m}\right]=(n-m) L_{n+m}+\frac{1}{12} \operatorname{Dn}\left(n^{2}-1\right) \delta_{n+m, 0},} \\
& {\left[L_{n}, \bar{L}_{m}\right]=0,} \\
& {\left[\bar{L}_{n}, \bar{L}_{m}\right]=(n-m) \bar{L}_{n+m}+\frac{1}{12} D n\left(n^{2}-1\right) \delta_{n+m, 0} .}
\end{aligned}
$$

Taking

$$
H=L_{0}+\bar{L}_{0}-2
$$

as a total hamiltonian, the commutation relation between $L_{n}(n>0)$ and $H$ reduces to the following form.

$$
\left[L_{n}, H\right]=n L_{n} \text {. }
$$

We can expand the above equation order by order in the number of weak fields

$$
\begin{aligned}
{\left[L_{n}^{(0)}, H^{(0)}\right] } & =n L_{n}^{(0)}, \\
{\left[L_{n}^{(0)}, H^{(1)}\right]+\left[L_{n}^{(1)}, H^{(0)}\right] } & =n L_{n}^{(1)}, \\
{\left[L_{n}^{(0)}, H^{(2)}\right]+\left[L_{n}^{(1)}, H^{(1)}\right]+\left[L_{n}^{(2)}, H^{(0)}\right] } & =n L_{n}^{(2)},
\end{aligned}
$$

etc.

Here the superscript $(i)$ denotes the number of weak fields which in general, on the contrary to eq. (2.5), include not only massless but also tachyon and massive fields. Let us consider the matrix element $T$

$$
T=\left\langle A\left|L_{n}^{(0)} S\right| B\right\rangle
$$

Here $|B\rangle$ satisfies the on-shell physical state conditions, eq. (2.20) and $\langle A|$ obeys

$$
\langle A|\left(H^{(0)}+n\right)=0
$$

but not necessarily the physical state condition.

At the first order, $T$ is given by (omitting an overall $2 \pi \delta(0)$ factor)

$$
\begin{aligned}
T^{(1)} & =\left\langle A\left|L_{n}^{(0)}\left(-i H^{(1)}\right)\right| B\right\rangle \\
& =(-i)\left\langle A\left|\left(H^{(1)} L_{n}^{(0)}+\left(H^{(0)}+n\right) L_{n}^{(1)}-L_{n}^{(1)} H^{(0)}\right)\right| B\right\rangle \\
& =0
\end{aligned}
$$


where we have used eq. (2.31) and in the final step on the on-shell physical state conditions for $|B\rangle$, and the on-shell condition for $|A\rangle$. We will next consider the second order term in the weak field expansion which we denote $T^{(2)}$. Using $\Delta=H^{(0)}$ as the inverse propagator, we have

$$
\begin{aligned}
T^{(2)}= & \left\langle A\left|L_{n}^{(0)}\left(-i H^{(2)}\right)\right| B\right\rangle \\
& +\left\langle A\left|L_{n}^{(0)}\left(-i H^{(1)}\right) \frac{(-i)}{\Delta}\left(-i H^{(1)}\right)\right| B\right\rangle \\
= & (-i)\left(\left\langle A\left|L_{n}^{(0)} H^{(2)}\right| B\right\rangle-\left\langle A\left|L_{n}^{(0)} H^{(1)} \frac{1}{\Delta} H^{(1)}\right| B\right\rangle\right) .
\end{aligned}
$$

The first term can be rewritten as follows:

$$
\begin{aligned}
\left\langle A\left|L_{n}^{(0)} H^{(2)}\right| B\right\rangle & =\left\langle A\left|\left(H^{(2)} L_{n}^{(0)}+(\Delta+n) L_{n}^{(2)}-L_{n}^{(2)} \Delta-\left[L_{n}^{(1)}, H^{(0)}\right]\right)\right| B\right\rangle \\
& =-\left\langle A\left|\left[L_{n}^{(1)}, H^{(1)}\right]\right| B\right\rangle
\end{aligned}
$$

where we have used eq. (2.32) and the on-shell and/or the physical state condition for $|B\rangle,\langle A|$ as before.

On the other hand, the second term reduces to the following form

$$
\begin{aligned}
-\left\langle A\left|L_{n}^{(0)} H^{(1)} \frac{1}{\Delta} H^{(1)}\right| B\right\rangle \\
=-\left\langle A\left|\left\{H^{(1)} L_{n}^{(0)}+(\Delta+n) L_{n}^{(1)}-L_{n}^{(1)} \Delta\right\} \frac{1}{\Delta} H^{(1)}\right| B\right\rangle \\
=-\left\langle A\left|H^{(1)} L_{n}^{(0)} \frac{1}{\Delta} H^{(1)}\right| B\right\rangle+\left\langle A\left|L_{n}^{(1)} H^{(1)}\right| B\right\rangle \\
=-\left\langle A\left|H^{(1)} \frac{1}{\Delta+n} L_{n}^{(0)} H^{(1)}\right| B\right\rangle+\left\langle A\left|L_{n}^{(1)} H^{(1)}\right| B\right\rangle \\
=-\left\langle A\left|H^{(1)} \frac{1}{\Delta+n}\left(H^{(1)} L_{n}^{(0)}+(\Delta+n) L_{n}^{(1)}-L_{n}^{(1)} \Delta\right)\right| B\right\rangle \\
\quad+\left\langle A\left|L_{n}^{(1)} H^{(1)}\right| B\right\rangle \\
=\left\langle A\left|\left[L_{n}^{(1)}, H^{(1)}\right]\right| B\right\rangle
\end{aligned}
$$


combining (2.30) and (2.35) we get

$$
T^{(2)}=0
$$

In this way, we can prove order by order in the weak field expansion that $T$ vanishes if we assume that the Virasoro algebra is satisfied. For completeness we will give the inductive proof to an arbitrary order in the weak field expansion in appendix $\mathrm{A}$. This establishes the equivalence of our unitarity constraint to the requirement of conformal symmetry.

\section{Explicit calculations of the matrix elements}

In the previous section, we have formulated the unitarity condition which states that the matrix element $T$ given by

$$
T=\left\langle A\left|L_{n}^{(0)} S\right| B\right\rangle
$$

should vanish for any choice of a physical state $|B\rangle$ and a state $\langle A|$ such as $\langle A| L_{n}^{(0)}$ is an on-shell state but not necessarily a physical state. Here $S$ is the $S$ matrix generated by the interaction hamiltonian

$$
S=T \exp \left(-i \int_{-\infty}^{\infty} H_{\mathrm{I}}\left(\phi_{\text {in }}\right) \mathrm{d} \tau\right) .
$$

The interaction hamiltonian contains contributions from the massless modes as well as the tachyon and other massive modes of the string.

We can now expand on the matrix element $T$ order by order in a weak field expansion, i.e.,

$$
T=T^{(1)}+T^{(2)}+\ldots,
$$

and demand that it vanish to each order in this weak field expansion. In this section we will explicitly show that the vanishing of $T^{(1)}$ gives rise to the free part of the equations of motion for the various modes, whereas the vanishing of $T^{(2)}$ gives the interaction terms in the equations of motion. In order to obtain this, we will use the weak-field expansion of the interaction hamiltonian,

$$
H_{\mathrm{I}}=H_{\text {massless }}^{(1)}+H_{\text {massless }}^{(2)}+\Delta H_{\text {tach }}^{(1)}+\Delta H_{1 \text { st massless }}^{(1)}+\ldots,
$$

where $H_{\text {massless }}^{(1)}, H_{\text {massless }}^{(2)}, \Delta H_{\text {tach }}^{(1)}$ and $\Delta H_{1 \mathrm{st} \text { massless }}^{(1)}$ are given in eqs. (2.8), (2.11), (2.17), (2.18). For the states $|B\rangle$ and $\langle A| L_{n}^{(0)}$ we will use the simplest non-trivial possibility that is consistent with the constraint $N=\tilde{N}$ for the matrix element. The state $|B\rangle$ is taken to be a tachyon state, i.e. $|B\rangle=|0, p\rangle$ with $p^{2}=8$ and the state $\langle A|$ is taken to be $\langle A|=\left\langle 0 p^{\prime}\right| \tilde{\alpha}_{1}^{\lambda}$. 
For the state $\langle A| L_{n}^{(0)}$, we will pick $n=1$ and then the condition that it be on shell, i.e., $\langle A| L_{n}^{(0)} H^{(0)},=0$, implies that $p^{\prime 2}=0$. Thus

$$
\langle A| L_{1}^{(0)}=p_{\sigma}^{\prime}\left\langle 0 P^{\prime}\right| \tilde{\alpha}_{1}^{\lambda}, \alpha_{1}^{\sigma} \quad \text { with } p^{\prime 2}=0 .
$$

In this form, we immediately recognize the state $\langle A| L_{1}^{(0)}$ to be that of a massless mode, with an unphysical polarization. Thus, with this choice of the stages, the unitarity condition will be seen to be essentially a Ward identity for the spacetime "gauge" invariance of the string theory.

In the next two subsections we will explicitly evaluate the matrix elements $T^{(1)}$ and $T^{(2)}$ and discuss how the equations of motion for the various string modes can be obtained by requiring that these vanish.

\subsection{FIRST ORDER CALCULATIONS}

We will begin with the evaluation of the contribution to $T^{(1)}$ for the massless modes (denoted by $T_{\text {massless }}^{(1)}$ ). The relevant term in the interaction hamiltonian is

$$
H_{\text {massless }}^{(1)}=\frac{1}{2 \pi} \int_{0}^{2 \pi} \mathrm{d} \sigma: \rho_{\mu \nu}(X) P^{\mu} \tilde{P}^{\nu}:
$$

Introducing the Fourier transform of $\rho_{\mu \nu}(X)$,

$$
: \rho_{\mu \nu}(X):=\int \frac{\mathrm{d}^{D} k}{(2 \pi)^{D}} \rho_{\mu \nu}(k): \mathrm{e}^{i k \cdot X}:
$$

we have

$$
H_{\text {massless }}^{(1)}=\int \frac{\mathrm{d}^{D} k}{(2 \pi)^{D}} \rho_{\mu \nu}(k) \frac{1}{2 \pi} \int_{0}^{2 \pi} \mathrm{d} \tau: \mathrm{e}^{i k \cdot X} P^{\mu} \tilde{P}^{\nu}:
$$

Omitting an overall factor of $2 \pi \delta(0)$, the expression for $T_{\text {massless }}^{(1)}$ becomes:

$$
\begin{aligned}
T_{\text {massless }}^{(1)} & =(-i)\left\langle A\left|L_{1}^{(0)} H_{\text {massless }}^{(1)}\right| B\right\rangle \\
& =(-i) \int \frac{\mathrm{d}^{D} k}{(2 \pi)^{D}} \rho_{\mu \nu}(k) p_{\boldsymbol{\sigma}}^{\prime}\left\langle 0, p^{\prime}\left|\tilde{\alpha}_{1}^{\lambda} \alpha_{1}^{\sigma} \frac{1}{2 \pi} \int_{0}^{2 \pi} \mathrm{d} \tau: \mathrm{e}^{i k \cdot X} P^{\mu} \tilde{P}^{\nu}:\right| 0, p\right\rangle .
\end{aligned}
$$

This can be readily evaluated using the standard methods used for calculating the string tree amplitudes and the result is;

$$
\begin{aligned}
T_{\text {massless }}^{(1)}= & (-i)\left\{\int \frac{\mathrm{d}^{D} k}{(2 \pi)^{D}}(2 \pi)^{D} \delta^{D}\left(p^{\prime}-p-k\right) \frac{1}{8} k^{2} \rho_{\mu \nu}(k)\right\} \\
& \times Y_{\text {massless }}^{\mu \nu, \lambda}\left(p, p^{\prime}\right),
\end{aligned}
$$


where, $Y_{\text {massless }}^{\mu \nu, \lambda}\left(p, p^{\prime}\right)$ is a tensor that depends on the external momenta, $p$ and $p^{\prime}$, i.e.,

$$
Y_{\text {massless }}^{\mu \nu, \lambda}\left(p, p^{\prime}\right)=\frac{1}{2}\left(\frac{p+p^{\prime}}{2}\right)^{\mu}\left(\eta^{\nu \lambda}+\frac{1}{2}\left(\frac{p^{\prime}+p}{2}\right)^{\nu}\left(\frac{p^{\prime}-p}{2}\right)^{\lambda}\right) .
$$

Because of this factorization property of $T_{\text {massless }}^{(1)}$, we see that if $T_{\text {massless }}^{(1)}$ is to vanish regardless of the values of $p, p^{\prime}$, then,

$$
k^{2} \rho_{\mu \nu}(k)=0 \text {. }
$$

In $x$-space, this gives the free part of the equations of motion, $\partial^{2} \rho_{\mu \nu}(x)=0$. The equations of motion for the graviton, the antisymmetric tensor and the dilation can be obtained by projecting out the appropriate spin projections of $\rho_{\mu \nu}(x)$.

As remarked earlier, with $n=1$ and the choice of states made above, the unitarity condition (3.1) is closely connected with the space-time Ward identity for "gauge" invariance. In general, the Ward identity states that for an $n$-point amplitude, if the polarization vector for one of the gravitons is replaced by its corresponding momenta, and all other particles are on shell, then the amplitude should vanish. However, in our case we require the vanishing of such an amplitude and get the on-shell conditions as a result.

The free part of the equations of motion for the other string modes may also be obtained in an analogous manner, by using the appropriate part of the interaction hamiltonian. For example, the expressions for $T_{\text {tach }}^{(1)}$ and $T_{\text {massive }}^{(1)}$ are:

$$
\begin{aligned}
T_{\mathrm{tach}}^{(1)} & =(-i)\left\langle A\left|L_{\mathrm{l}}^{(0)} \Delta H_{\mathrm{tach}}^{(1)}\right| B\right\rangle, \\
T_{\text {massive }}^{(1)} & =(-i)\left\langle A\left|L_{1}^{(0)} \Delta H_{1 \mathrm{st} \text { massive }}^{(1)}\right| B\right\rangle,
\end{aligned}
$$

with $\Delta H_{\text {tach }}^{(1)}$ and $\Delta H_{1 \text { st massive }}^{(1)}$ given in (2.17) and (2.18).

For the first massive excitation $M_{\mu \alpha, \nu \beta}(X)$ we will assume the gauge conditions (2.19). We may introduce the Fourier transforms

$$
\begin{gathered}
: \phi(X):=\int \frac{\mathrm{d}^{D} k}{(2 \pi)^{D} \phi(k): \mathrm{e}^{i k \cdot X}:}, \\
: M_{\mu \alpha, \nu \beta}(X):=\int \frac{\mathrm{d}^{D} k}{(2 \pi)^{D}} M_{\mu \alpha, \nu \beta}(k): \mathrm{e}^{i k \cdot X}:,
\end{gathered}
$$


and then the matrix elements are readily evaluated. The results are (omitting and over $2 \pi \delta(0))$ :

$$
\begin{aligned}
& T_{\text {tach }}^{(1)}=(-i)\left\{\int \frac{\mathrm{d}^{D} k}{(2 \pi)^{D}}(2 \pi)^{D} \delta^{D}\left(p^{\prime}-p-k\right)\left(\frac{1}{8} k^{2}-1\right) \phi(k)\right\} Y_{\text {tach }}^{\lambda}, \\
& Y_{\text {tach }}^{\lambda}=\frac{1}{2}\left(p^{\prime}-p\right)^{\lambda},
\end{aligned}
$$

and

$$
\begin{aligned}
T_{\text {massive }}^{(1)}= & (-i)\left\{\int \frac{\mathrm{d}^{D} k}{(2 \pi)^{D}}(2 \pi)^{D} \delta\left(p^{\prime}-p-k\right)\left(\frac{1}{8} k^{2}+1\right) M_{\mu \alpha \nu \beta}(k)\right\} \\
& \times Y_{\text {massive }}^{\mu \alpha, \nu \beta},
\end{aligned}
$$

with

$$
\begin{aligned}
Y_{\text {massive }}^{\mu \alpha, \nu \beta}= & \left.\frac{\left(p+p^{\prime}\right)^{\mu}}{4} \frac{\left(p+p^{\prime}\right)^{\alpha}}{4}\right) \\
& \times\left[\eta^{\lambda \nu} \frac{p^{\beta}}{2}+\eta^{\lambda \beta} \frac{p^{\nu}}{2}+\left(\frac{p^{\prime}-p}{2}\right)^{\lambda}\left(\frac{p+p^{\prime}}{4}\right)^{\nu}\left(\frac{p+p^{\prime}}{4}\right)^{\beta}\right] .
\end{aligned}
$$

The amplitudes $T_{\text {tach }}^{(1)}, T_{\text {massive }}^{(1)}$ have the same factionized form as $T_{\text {massive }}^{(1)}$ and the tensor structures, $Y_{\text {massless, }}^{\mu \nu, \lambda}, Y_{\text {tach }}^{\lambda}, Y_{\text {massive }}^{\mu \alpha, \nu \beta}$ are independent, hence by requiring these to vanish, we get the free part of the equation of motion for the different modes. For the tachyon, in $x$-space this is

$$
\left(\partial^{2}+8\right) \phi(x)=0
$$

and for the first massive excitation, the equation of motion in the gauge, $\partial^{\mu} M_{\mu \alpha \nu \beta}=$ $\partial^{\alpha} M_{\mu \alpha \nu \beta}=\partial^{\nu} M_{\mu \alpha \nu \beta}=\partial^{\beta} M_{\mu \alpha \nu \beta}=0 ; \eta^{\mu \alpha} M_{\mu \alpha \nu \beta}=\eta^{\nu \beta} M_{\mu \alpha \nu \beta}=0$, is

$$
\left(\partial^{2}-8\right) M_{\mu \alpha \nu \beta}=0
$$

We will next consider the second order term in the weak field expansion of $T$. When combined with the above first order results, we will see that we obtain the interaction terms in the equations of motion for the various modes. 


\subsection{SECOND ORDER CALCULATIONS}

We will now consider the second order term in the weak field expansion of the matrix element $T$. (This will be denoted by $T^{(2)}$ ). In general, $T^{(2)}$ has many components: there will be terms of second order in $\rho_{\mu \nu}(x)$ (denoted by $T_{\rho 2}^{(2)}$ ), terms of second order in $\phi(x)$ (denoted by $T_{\phi 2}^{(2)}$ ), cross-terms which are first order in $\rho_{\mu \nu}(x)$ and $\phi(x)$ (denoted by $\left.T_{\rho \phi}^{(2)}\right)$, terms which are second order in $M_{\mu \alpha \nu \beta}(x)$, and so on, i.e.,

$$
T^{(2)}=T_{\rho 2}^{(2)}+T_{\phi 2}^{(2)}+T_{\rho \phi}^{(2)}+\ldots
$$

In this paper, we will only consider the contributions $T_{\rho 2}^{(2)}, T_{\phi 2}^{(2)}$ and $T_{\rho \phi}^{(2)}$. We will first write down the explicit forms of these and then discuss how the interaction parts of the equations of motion may be extracted. The techniques used for the evaluation of these matrix elements are similar to those for calculating the standard string tree amplitudes [5]. To illustrate the method we will first discuss the evaluation of the matrix element $T_{\rho \phi}^{(2)}$ in some detail.

The matrix element $T_{\rho \phi}^{(2)}$ is given by

$$
\begin{aligned}
& T_{\rho \phi}^{(2)}=(-i)^{3}\left\{\left\langle A\left|L_{1}^{(0)} H_{\text {massless }}^{(1)}(0) \frac{1}{\Delta} \Delta H_{\text {tach }}^{(1)}(0)\right| B\right\rangle\right. \\
&\left.+\left\langle A\left|L_{1}^{(0)} \Delta H_{\text {tach }}^{(1)}(0) \frac{1}{\Delta} H_{\text {massless }}^{(1)}(0)\right| B\right\rangle\right\} \\
&=(-i)^{3}\left(\frac{p^{\prime}}{2}\right)^{o}\left\{\left\langle 0, p^{\prime}\right| \tilde{\alpha}_{1}^{\lambda} \alpha_{1}^{\sigma} \int \frac{\mathrm{d} \sigma_{1}}{2 \pi} \int \frac{\mathrm{d}^{D} k_{1}}{(2 \pi)^{D}} \rho_{\mu \nu}\left(k_{1}\right)\right. \\
& \times: \mathrm{e}^{i k_{1} \cdot X\left(\sigma_{1}\right)} P_{\left(\sigma_{1}\right)}^{\mu} \tilde{P}_{\left(\sigma_{1}\right)}^{\nu}: \int_{0}^{1} \frac{\mathrm{d} y}{y} y^{\Delta} \int \frac{\mathrm{d} \sigma_{2}}{2 \pi} \int \frac{\mathrm{d}^{D} k}{(2 \pi)^{D}} \phi\left(k_{2}\right): \mathrm{e}^{i k_{2} \cdot X\left(\sigma_{2}\right):|0, p\rangle} \\
&+\left\langle 0, p^{\prime}\right| \tilde{\alpha}_{1}^{\lambda} \alpha_{1}^{\sigma} \int \frac{\mathrm{d} \sigma_{2}}{(2 \pi)} \int \frac{\mathrm{d}^{D} k_{2}}{(2 \pi)^{D}} \phi\left(k_{2}\right): \mathrm{e}^{i k_{2} \cdot X\left(\sigma_{2}\right)}: \\
& \times \int_{0}^{1} \frac{\mathrm{d} y}{y} y^{\Delta} \int \frac{\mathrm{d} \sigma_{1}}{2 \pi} \int \frac{\mathrm{d}^{D} k_{1}}{\left.(2 \pi)^{D} \rho_{\mu \nu}\left(k_{1}\right): \mathrm{e}^{i k_{1} \cdot X\left(\sigma_{1}\right)} P^{\mu}\left(\sigma_{1}\right) \tilde{P}^{\nu}\left(\sigma_{1}\right):|0, p\rangle\right\},},
\end{aligned}
$$


where, as before, we have introduced the Fourier transform of $\rho_{\mu \nu}(x)$ and $\phi(x)$ and used the representation

$$
\frac{1}{\Delta}=\int_{0}^{1} \frac{\mathrm{d} y}{y} y^{\Delta}
$$

We may now use the shift operations for any operator $O(\sigma, \tau)$, i.e.

$$
\begin{aligned}
& O(\sigma, \tau)=\mathrm{e}^{i H_{0} \tau} O(\sigma, 0) \mathrm{e}^{-i H_{0} \tau}, \\
& O(\sigma, \tau)=\mathrm{e}^{-i\left(L_{0}-\bar{L}_{0}\right) \sigma} O(0, \tau) \mathrm{e}^{i\left(L_{0}-\bar{L}_{0}\right) \sigma},
\end{aligned}
$$

to rewrite (3.23) as a function only of $\sigma_{2}-\sigma_{1}$, and $\tau=i l n y$. Then,

$$
\begin{aligned}
T_{\rho \phi}^{(2)}=( & -i)^{3}\left(\frac{p^{\prime}}{2}\right)^{\sigma} \int \frac{\mathrm{d}^{D} k_{1}}{(2 \pi)^{D}} \int \frac{\mathrm{d}^{D} k_{2}}{(2 \pi)^{D}} \rho_{\mu \nu}\left(k_{1}\right) \phi\left(k_{2}\right) \int \frac{\mathrm{d} \sigma}{2 \pi} \\
& \times\left\{\int_{1}^{\infty} \frac{\mathrm{d} y}{y}\left\langle 0, p^{\prime}\right| \tilde{\alpha}_{1}^{\lambda} \alpha_{1}^{\sigma}\right. \\
& \times: \mathrm{e}^{i k_{1} X(0,0)} P^{\mu}(0,0) \tilde{P}^{\nu}(0,0):: \mathrm{e}^{i k_{2} X(\sigma, r=i l n y)}:|0, p\rangle \\
+ & \left.\int_{0}^{1} \frac{\mathrm{d} y}{y}\left\langle 0, p^{\prime} \mid \tilde{\alpha}_{1}^{\lambda} \alpha_{1}^{\sigma}: \mathrm{e}^{i k_{2} X(\sigma, \tau=i(n y)}:: \mathrm{e}^{i k_{1} x(0,0)} P^{\mu}(0) \tilde{P}^{\nu}(0): 0, p\right\rangle\right) .
\end{aligned}
$$

We next let $Z=y \mathrm{e}^{i \sigma}$, and

$$
\begin{aligned}
X_{\mu}(Z, \bar{Z}) & =x^{\mu}+\frac{1}{2} p_{\mu}(i \ln |Z|)+\frac{1}{2} i \sum_{n \neq 0} \frac{1}{n}\left(\alpha_{n \mu} Z^{n}+\tilde{\alpha}_{n \mu} \bar{Z}^{n}\right) \\
& \equiv x_{\mu}|Z, \bar{Z}|+\frac{1}{2} p_{\mu}(i \ln |Z|) .
\end{aligned}
$$

Then, using

$$
\begin{gathered}
\mathrm{e}^{i k_{2} \cdot X(Z, \bar{Z})}=\mathrm{e}^{i k_{2} x(Z, \bar{Z})}|Z|^{\frac{1}{2}\left(p \cdot k_{2}\right)-\frac{1}{4} k_{2}^{\frac{2}{2}}}, \\
|Z|^{-\frac{1}{2}\left(p \cdot k_{2}\right)} \mathrm{e}^{i k_{1} \cdot X}=\mathrm{e}^{i k_{1} \cdot X}|Z|^{-\frac{1}{2}\left(p \cdot k_{2}\right)-\frac{1}{2}\left(k_{1} \cdot k_{2}\right)},
\end{gathered}
$$


we can use the matrix element as:

$$
\begin{aligned}
T_{\rho \phi}^{(2)}= & \frac{(-i)^{3}}{2 \pi}\left(\frac{p^{\prime}}{2}\right)^{\sigma} \int \frac{\mathrm{d}^{D} k_{1}}{(2 \pi)^{D}} \int \frac{\mathrm{d}^{D} k_{2}}{(2 \pi)^{D}} \rho_{\mu \nu}\left(k_{1}\right) \phi\left(k_{2}\right) \\
& \times \int_{|Z|\rangle \mid} \frac{\mathrm{d}^{2} Z}{|Z|^{2}}|Z|^{-\frac{1}{2}\left(p \cdot k_{2}\right)-\frac{1}{4} k_{2}^{2}} \\
& \times\left\langle 0, p^{\prime}\left|\tilde{\alpha}_{1}^{\lambda} \alpha_{1}^{\sigma}: \mathrm{e}^{i k_{1} X(1)} P^{\mu}(1) \tilde{P}^{\nu}(1):: \mathrm{e}^{i k_{2} X(Z, \bar{Z})}:\right| 0, p\right\rangle \\
& +\int_{|Z|\langle 1} \frac{\mathrm{d}^{2} Z}{|Z|^{2}}|Z|^{-\frac{1}{2}\left(p \cdot k_{2}\right)-\frac{1}{4} k_{2}^{2}-\frac{1}{2} k_{1} k_{2}}\left\langle 0, p^{\prime}\right| \tilde{\alpha}_{1}^{\lambda} \alpha_{1}^{\sigma}: \\
& \left.\times \mathrm{e}^{i k_{2} X(Z, \bar{Z})}:: \mathrm{e}^{i k_{1} X(1)} P^{\mu}(1) \tilde{P}^{\nu}(1):|0, p\rangle\right\},
\end{aligned}
$$

we may now perform the contractions using the standard formulae given, for example, in ref. [4], to get

$$
\begin{aligned}
& : \mathrm{e}^{i k_{1} x(1)} P^{\mu}(1) \tilde{P}^{\nu}(1):: \mathrm{e}^{i k_{2} x(Z, \bar{Z})}:(|Z|>1) \\
& =\left|1-\frac{1}{Z}\right|^{\frac{1}{2}\left(k_{1} \cdot k_{2}\right)}\left\{: \mathrm{e}^{i k_{1} x(1)+i k_{2} x(Z, \bar{Z})} P^{\mu}(1) \tilde{P}^{\nu}(1)\right. \text { : } \\
& +\frac{k_{2}^{\nu}}{2} \frac{Z}{Z-1}: \mathrm{e}^{i k_{1} x(1)+i k_{2} x(Z, \bar{Z})} \tilde{P}^{\nu}(0): \\
& +\frac{k_{2}^{\nu}}{2} \frac{\bar{Z}}{\bar{Z}-1}: \mathrm{e}^{i k_{1} x(1)+i k_{1} x(Z, \bar{Z})} P^{\mu}(1): \\
& \left.+\frac{k_{2}^{\mu}}{2} \frac{k_{2}^{\nu}}{2} \frac{|Z|^{2}}{|Z-1|^{2}}: \mathrm{e}^{i k_{1} x(1)+i k_{2} x(Z, \bar{Z})}:\right\} \\
& \times: \mathrm{e}^{i k_{2} X(Z . \bar{z})}:: \mathrm{e}^{i k_{1} x(1)} P^{\mu}(1) \tilde{P}^{\nu}(1):(|Z|\langle 1) \\
& =|1-Z|^{\frac{1}{2}\left(k_{1} \cdot k_{2}\right)}\left\{: \mathrm{e}^{i k_{1} x(1)+i k_{2} x(Z, \bar{Z})} P^{\mu}(1) \tilde{P}^{\nu}(1):\right. \\
& +\frac{k_{2}^{\mu}}{2} \frac{Z}{Z-1}: \mathrm{e}^{i k_{1} x(1)+i k_{2} x(Z, \bar{Z})} \tilde{P}^{\nu}(1): \\
& +\frac{k_{2}^{\nu}}{2} \frac{\bar{Z}}{\bar{Z}-1}: \mathrm{e}^{i k_{1} x(1)+i k_{2} x(Z, \bar{Z})} P^{\mu}(1) \text { : } \\
& \left.+\frac{k_{2}^{\mu}}{2} \frac{k_{2}^{\nu}}{2} \frac{|Z|^{2}}{|Z-1|^{2}}: \mathrm{e}^{i k_{1} x(1)+i k_{2} x(Z, \bar{Z})}:\right\}
\end{aligned}
$$


Upon inserting these into (3.29), we may easily combine the two terms to get an integral of $Z$ over the whole $Z$-plane to get:

$$
\begin{aligned}
& T_{\rho \phi}^{(2)}= \frac{(-i)^{3}}{(2 \pi)}\left(\frac{p^{\prime}}{2}\right)^{\sigma} \int \frac{\mathrm{d}^{D} k_{1}}{(2 \pi)^{D}} \\
& \times \int \frac{\mathrm{d}^{D} k_{2}}{(2 \pi)^{D}} \rho_{\mu \nu}\left(k_{1}\right) \phi\left(k_{2}\right) \\
& \times \int \frac{\mathrm{d}^{2} Z}{|Z|^{2}}|Z|^{-\frac{1}{2}\left(p \cdot k_{2}\right)-\frac{1}{4} k_{2}^{2}-\frac{1}{2}\left(k_{1} \cdot k_{2}\right)} \\
& \times|1-Z|^{\frac{1}{2}\left(k_{1} \cdot k_{2}\right)}\left\{\langle 0 , p ^ { \prime } | \tilde { \alpha } _ { 1 } ^ { \lambda } \alpha _ { 1 } ^ { \sigma } \left[: \mathrm{e}^{i k_{1} X(1)+i k_{2} X(Z, \bar{Z})} P^{\mu}(1) \tilde{P}^{\nu}(1):\right.\right. \\
& \quad+\frac{k_{2}^{\mu}}{2} \frac{Z}{Z-1}: \mathrm{e}^{i k_{1} X(1)+i k_{2} X(Z, \tilde{Z})} \tilde{P}^{\nu}(1): \\
& \quad+\frac{k_{1}^{\mu}}{2} \frac{k_{2}^{\nu}}{2} \frac{\mid \bar{Z}}{|Z|^{2}}: \mathrm{e}^{i k_{1} X(1)+i k_{2} X(Z, \bar{Z})} P^{\nu}(1): \\
&\left.|Z|^{i k_{1} X(1)+i k_{2} X(Z, \bar{Z})}:|0 \cdot p\rangle\right\} .
\end{aligned}
$$

The matrix element inside \{ $\}$ can be readily evaluated after moving the oscillators $\tilde{\alpha}^{\lambda}$ and $\alpha_{1}^{\sigma}$ to the extreme right using the commutation relations and we get after some algebra:

$$
\begin{aligned}
T_{\rho \phi}^{(2)}= & \frac{(-i)^{3}}{(2 \pi)}\left(\frac{p^{\prime}}{2}\right)^{\sigma} \int \frac{\mathrm{d}^{D} k_{1}}{(2 \pi)^{D}} \int \frac{\mathrm{d}^{D} k_{2}}{(2 \pi)^{D}} \rho_{\mu \nu}\left(k_{1}\right) \phi\left(k_{2}\right) \int \frac{\mathrm{d}^{2} Z}{|Z|^{2}} \\
& \times|Z|^{-\frac{1}{2}\left(p \cdot k_{2}\right)-\frac{1}{4} k_{2}^{2}-\frac{1}{2}\left(k_{1} \cdot k_{2}\right)}|1-Z|^{\frac{1}{2}\left(k_{1} \cdot k_{2}\right)}(2 \pi)^{D} \delta^{D}\left(p^{\prime}-k_{1}-k_{2}-p\right) \\
& \times\left\{\left(\frac{1}{2} k_{1}^{\sigma}+\frac{1}{2} k_{2}^{\sigma} Z^{-1}\right)\left(\frac{1}{2} k_{1}^{\lambda}+\frac{1}{2} k_{2}^{\lambda} \bar{Z}^{-1}\right) \frac{1}{2} p^{\mu \frac{1}{2} p^{\nu}}\right. \\
& +\left(\frac{1}{2} k_{1 \sigma}+\frac{1}{2} k_{2}^{\sigma} Z^{-1}\right)\left(\eta^{\lambda \nu}+\frac{k_{2}^{\nu}}{2} \frac{\bar{Z}}{\bar{Z}-1}\left(\frac{1}{2} k_{1}^{\lambda}+\frac{1}{2} k_{2}^{\lambda} \bar{Z}^{-1}\right)\right) \frac{1}{2} p^{\mu} \\
& \times\left(\frac{1}{2} k_{1}^{\lambda}+\frac{1}{2} k_{2}^{\lambda} \bar{Z}^{-1}\right)\left(\eta^{\sigma \nu}+\frac{k_{2}^{\mu}}{2} \frac{Z}{Z-1}\left(\frac{1}{2} k_{1}^{\sigma}+\frac{1}{2} k_{2}^{\sigma} Z^{-1}\right)\right) \frac{1}{2} p^{\nu} \\
& +\left[\eta^{\lambda \nu}\left(\eta^{\sigma \mu}+\frac{k_{2}^{\mu}}{2} \frac{Z}{Z-1}\left(\frac{1}{2} k_{1}^{\sigma}+\frac{1}{2} k_{2}^{\sigma} Z^{-1}\right)\right)\right. \\
& \left.\left.+\left(\frac{1}{2} k_{1}^{\lambda}+\frac{1}{2} k_{2}^{\lambda} \bar{Z}^{-1}\right)\left(\frac{k_{2}^{\nu}}{2} \frac{\bar{Z}}{\bar{Z}-1} \eta^{\sigma \mu}+\frac{k_{2}^{\mu}}{2} \frac{k_{2}^{\nu}}{2} \frac{|Z|^{2}}{|Z-1|^{2}}\left(\frac{1}{2} k_{1}^{\sigma}+\frac{1}{2} k_{2}^{\sigma} Z^{-1}\right)\right)\right]\right\} .
\end{aligned}
$$


The $Z$ integrations can be performed using the integral formulae of appendix $C$. The final result after contraction with $\left(\frac{1}{2} p^{\prime}\right)^{\sigma}$ is given by:

$$
\begin{aligned}
T_{\rho \phi}^{(2)}= & \frac{(-i)^{3}}{2} \int \frac{\mathrm{d}^{D} k_{1}}{(2 \pi)^{D}} \int \frac{\mathrm{d}^{D} k_{2}}{(2 \pi)^{D}} \rho_{\mu \nu}\left(k_{1}\right) \phi\left(k_{2}\right)(2 \pi)^{D} \delta^{D}\left(p^{\prime}-k_{1}-k_{2}-p\right) \\
& \times\left\{\frac{\tilde{t}}{8}\left(\left(\frac{s}{8}+1\right) \frac{k_{1}^{2}}{8}-\left(\frac{u}{8}+1\right)\left(\frac{k_{2}^{2}}{8}-1\right)\right) \frac{p^{\mu}}{2}\right. \\
& \left.-\left(\frac{s}{8}+1\right)\left(\frac{s}{8} \frac{k_{1}^{2}}{8}-\left(\frac{u}{8}+1\right)\left(\frac{k_{2}^{2}}{8}-1\right)\right) \frac{k_{2}^{\mu}}{2}\right\} \\
& \times\left\{\frac { \tilde { t } } { 8 } \frac { p ^ { \nu } } { 2 } \left(\frac{k_{1}^{\lambda}}{2}\left(\frac{s}{8}+1\right)-\frac{k_{2}^{\lambda}}{2}\left(\frac{u}{8}+1\right)\right.\right. \\
& \left.\quad-\left(\frac{s}{8}+1\right) \frac{k_{2}^{\nu}}{2}\left(\frac{k_{1}^{\lambda}}{2} \frac{s}{8}-\frac{k_{2}^{\lambda}}{2}\left(\frac{u}{8}+1\right)\right)+\frac{\tilde{t}}{8}\left(\frac{s}{8}+1\right) \eta^{\lambda \nu}\right\} \\
& \frac{\Gamma\left(-\frac{s}{8}-1\right) \Gamma\left(-\frac{u}{8}-1\right) \Gamma\left(-\frac{t}{8}-1-\frac{1}{8}\left(k_{1}^{2}+k_{2}^{2}-8\right)\right)}{\Gamma\left(\frac{s}{8}+2\right) \Gamma\left(\frac{u}{8}+2\right) \Gamma\left(\frac{t}{8}+\frac{1}{8}\left(k_{1}^{2}+k_{2}^{2}-8\right)+2\right)}
\end{aligned}
$$

Here, $s, t, u$ are the kinematic invariants defined by

$$
\begin{array}{ll}
s=-\left(p+k_{2}\right)^{2}, & t=-\left(p^{\prime}-p\right)^{2}=-\left(k_{1}+k_{2}\right)^{2}, \\
u=-\left(p+k_{2}\right)^{2}, \quad \tilde{t}=t+k_{1}^{2}+k_{2}^{2} .
\end{array}
$$

In writing down the expression, we have used $k_{1}^{\mu} \rho_{\mu \nu}\left(k_{1}\right)=0$ and the on-shell conditions for the external states, i.e., $p^{\prime 2}=0, p^{2}=8$.

The matrix elements $T_{\phi 2}^{(2)}$ and $T_{\rho 2}^{(2)}$ can be similarly evaluated. Consider $T_{\phi 2}^{(2)}$ next:

$$
T_{\phi 2}^{(2)}=(-i)^{3}\left\langle A\left|L_{1}^{(0)} \Delta H_{\mathrm{tach}}^{(1)}(0) \frac{1}{\Delta} \Delta H_{\mathrm{tach}}^{(1)}(0)\right| B\right\rangle
$$

Since this can be evaluated in a similar manner as the $T_{\rho \phi}^{(2)}$ term, we will just quote 
the final result:

$$
\begin{aligned}
T_{\phi 2}^{(2)}= & \frac{(-i)^{3}}{4} \int \frac{\mathrm{d}^{D} k_{1}}{(2 \pi)^{D}} \int \frac{\mathrm{d}^{D} k_{2}}{(2 \pi)^{D}} \phi\left(k_{1}\right) \phi\left(k_{2}\right)(2 \pi)^{D} \delta^{D}\left(p^{\prime}-k_{1}-k_{2}-p\right) \\
& \times\left(\frac{\tilde{t}}{8}\right)^{2}\left(1+\frac{\tilde{t}}{8}\right)^{2}\left\{\frac{k_{1}^{\lambda}}{2}\left[\left(\frac{k_{2}^{2}}{8}-1\right)\left(\frac{u}{8}+1\right)-\left(\frac{k_{1}^{2}}{8}-1\right)\left(\frac{s}{8}+1\right)\right]\left(\frac{s}{8}+1\right)\right. \\
& \left.+\frac{k_{2}^{\lambda}}{2}\left[\left(\frac{k_{1}^{2}}{8}-1\right)\left(\frac{s}{8}+1\right)-\left(\frac{k_{2}^{2}}{8}-1\right)\left(\frac{u}{8}+1\right)\right]\left(\frac{u}{8}+1\right)\right\} \\
& \times \Gamma\left(-\frac{u}{8}-1\right) \Gamma\left(-\frac{s}{8}-1\right) \Gamma\left(-\frac{\tilde{t}}{8}-1\right) / \Gamma\left(\frac{s}{8}+2\right) \Gamma\left(\frac{u}{8}+2\right) \Gamma\left(\frac{\tilde{t}}{8}+2\right)
\end{aligned}
$$

Finally, we consider the term $T_{\rho 2}^{(2)}$, which is second order in $\rho_{\mu \nu}(x)$. This has two contributions, and is given by

$$
T_{\rho 2}^{(2)}=(-i)^{3}\left\{\left\langle A\left|L_{1}^{(0)} H_{\text {massless }}^{(1)} \frac{1}{\Delta} H_{\text {massless }}^{(1)}\right| B\right\rangle-\left\langle A\left|L_{1}^{(0)} H_{\text {massless }}^{(2)}\right| B\right\rangle\right\}
$$

Consider the second term on the right hand side of (3.38). This term is analyzed in appendix $B$ to which the reader is referred to for details. The main point here is that in theories with derivative interactions, one should carefully distinguish between the $\mathrm{T}$ and $\mathrm{T}^{*}$ products. Contractions using the $\mathrm{T}$ product and the $\mathrm{T}^{*}$ product are not the same. In the hamiltonian formulation, one has to use the $T$ product and the difference between this and the $\mathrm{T}^{*}$ product is cancelled by the second term in (3.38) in accordance with the Nambu-Mathews theorem. The standard string operator formalism, which we are using here, corresponds to the $\mathrm{T}^{*}$ product and therefore we drop this term henceforth from our consideration. Thus, with the provision that we use the $\mathrm{T}^{*}$ product,

$$
T_{\rho 2}^{(2)}=(-i)^{3}\left\langle A\left|L_{1}^{(0)} H_{\text {massless }}^{(1)} \frac{1}{\Delta} H_{\text {massless }}^{(1)}\right| B\right\rangle
$$


The evaluation of this is similar to that of $T_{\rho \phi}^{(2)}$ and the result is,

$$
\begin{aligned}
T_{\rho 2}^{(2)}= & -\frac{(-i)^{3}}{16} \int \frac{\mathrm{d}^{D} k_{1}}{(2 \pi)^{D}} \int \frac{\mathrm{d}^{D} k_{2}}{(2 \pi)^{D}} \rho_{\mu \nu}\left(k_{1}\right) \rho_{\alpha \beta}\left(k_{2}\right)(2 \pi)^{D} \delta^{D}\left(p^{\prime}-k_{1}-k_{2}-p\right) \\
& \times J^{\mu \alpha} K^{\lambda \nu \beta} \frac{\Gamma\left(-1-\frac{s}{8}\right) \Gamma\left(-1-\frac{u}{8}\right) \Gamma\left(-1-\frac{\tilde{t}}{8}\right)}{\Gamma\left(2+\frac{s}{8}\right) \Gamma\left(2+\frac{u}{8}\right) \Gamma\left(2+\frac{\tilde{t}}{8}\right)}
\end{aligned}
$$

Here, $s, t, u$, are the kinematic invariants and $K^{\lambda \nu \beta}$ and $J^{\mu \alpha}$ are given by:

$$
\begin{aligned}
K^{\lambda \nu \beta}= & \frac{\tilde{t}}{8}\left(1+\frac{\tilde{t}}{8}\right)\left[\frac{p^{\beta}}{2}\left(\eta^{\lambda \nu}+\frac{p^{\nu} k_{1}^{\beta}}{4}\right)\left(2+\frac{s}{4}\right)-\frac{p^{\nu}}{2}\left(\eta^{\lambda \beta}+\frac{p^{\beta} k_{2}^{\lambda}}{4}\left(2+\frac{u}{4}\right)\right]\right. \\
& +2\left(1+\frac{\tilde{t}}{8}\right)\left(1+\frac{u}{8}\right)\left(1+\frac{s}{8}\right)\left[\frac{k_{2}^{\nu}}{2}\left(\eta^{\lambda \beta}+\frac{k_{2}^{\lambda}}{4} p^{\beta}\right)-\frac{k_{1}^{\beta}}{2}\left(\eta^{\lambda \nu}+\frac{k_{1}^{\lambda} p^{\nu}}{4}\right)\right] \\
& +\left(1+\frac{\tilde{t}}{8}\right)\left[\frac{k_{2}^{\lambda}}{2} \frac{k_{1}^{\beta}}{2} \frac{p^{\nu}}{2} \frac{u}{4}\left(1+\frac{u}{8}\right)-\frac{k_{1}^{\lambda}}{2} \frac{k_{2}^{\nu}}{2} \frac{p^{\beta}}{2} \frac{s}{4}\left(1+\frac{s}{8}\right)\right] \\
& +\left(\frac{k_{1}^{\beta} k_{2}^{\nu}}{4}-\eta^{\beta \nu}\right)\left(\frac{k_{1}^{\lambda}}{2} \frac{s}{4}-\frac{k_{2}^{\lambda}}{2} \frac{u}{4}\right)\left(1+\frac{s}{8}\right)\left(1+\frac{u}{8}\right) \\
J^{\mu \alpha}= & \left(\frac{p^{\prime}}{2}\right) K_{\sigma}^{\sigma \mu \alpha} \\
= & \frac{\tilde{t}}{8}\left(1+\frac{\tilde{t}}{8}\right)\left[\left(2+\frac{s}{4}\right) \frac{k_{1}^{2}}{8}-\left(2+\frac{u}{4}\right) \frac{k_{2}^{2}}{8}\right] \frac{p^{\mu}}{2} \frac{p^{\alpha}}{2} \\
& +2\left(1+\frac{s}{8}\right)\left(1+\frac{\tilde{t}}{8}\right)\left[\left(1+\frac{u}{8}\right) \frac{k_{2}^{2}}{8}-\frac{s}{8} \frac{k_{1}^{2}}{8}\right] \frac{k_{2}^{\mu}}{2} \frac{p^{\alpha}}{2} \\
& +2\left(1+\frac{u}{8}\right)\left(1+\frac{\tilde{t}}{8}\right)\left[-\left(1+\frac{s}{8}\right) \frac{k_{2}^{2}}{8}+\frac{u}{8} \frac{k_{1}^{2}}{8}\right] \frac{k_{1}^{\alpha}}{2} \frac{p^{\mu}}{2} \\
& +\left(\frac{k_{1}^{\alpha} k_{2}^{\mu}}{4}-\eta^{\alpha \nu}\right)\left[\frac{s}{4} \frac{k_{1}^{2}}{8}-\frac{u}{4} \frac{k_{2}^{2}}{8}\right]\left(1+\frac{s}{8}\right)\left(1+\frac{u}{8}\right) \\
& (3.42) \\
& (3.4) \\
&
\end{aligned}
$$


In the above expression we have used the gauge conditions $k_{1}^{\mu} \rho_{\mu \nu}\left(k_{1}\right)=k_{2}^{\alpha} \rho_{\alpha \beta}\left(k_{2}\right)$ $=0$ and the on-shell conditions, $p^{\prime 2}=0, p^{2}=8$ for the external states.

We will now discuss how the $\rho \rho, \rho \phi$ and $\phi \phi$ parts of the equations of motion for the different modes may be obtained.

First, we note that the expressions (3.34), (3.37) and (3.40) for $T_{\rho \phi}^{(2)}, T_{\phi 2}^{(2)}$ and $T_{\rho 2}^{(2)}$ vanish when we use the first order result. For example, $T_{\rho \phi}^{(2)}$ vanishes upon using $k_{1}^{2} \rho_{\mu \nu}\left(k_{2}\right)=0,\left(k_{2}^{2}-8\right) \phi\left(k_{2}\right)=0 ; T_{\phi 2}^{(2)}$ vanishes upon using $\left(k_{1}^{2}-8\right) \phi\left(k_{1}\right)=0$ and $\left(k_{2}^{2}-8\right) \phi\left(k_{2}\right)=0$ and $T_{\rho 2}^{(2)}$ vanishes if we use $k_{1}^{2} \rho_{\mu \nu}(k)=k_{2}^{2} \rho_{\mu \nu}\left(k_{2}\right)=0$. This is a consequence of the gauge invariance of the massless modes, and corresponds to the space-time Ward identity of the 4-point function alluded to earlier. However, non-trivial contributions arise when we set $t$ equal to one of the on-mass shell values, i.e., whenever,

$$
\frac{t}{8}=-\frac{\left(p^{\prime}-p\right)^{2}}{8}=-1,0,1,2, \ldots
$$

Then, the corresponding gamma-functions, in the $t$ variable in $T_{\rho \phi}^{(2)}$, in $T_{\phi 2}^{(2)}$ and in $T_{\rho 2}^{(2)}$ develop a $t$-channel pole and the above expression becomes of the indeterminate $0 / 0$ form. These contributions can be extracted by using a proper infrared regularization and as we shall see, they correspond to the interaction terms in the equations of motion. For $t \sim 0$ and $t \sim 8$ we will get contributions to the massless and tachyon equations. The infrared regularization we adopt here corresponds to shifting the mass-shell condition for $\rho_{\mu \nu}\left(k\left({ }_{1}\right), \phi\left(k_{2}\right)\right.$, or $\rho_{\mu \nu}\left(k_{2}\right)$ by the same amount, i.e., we let $\left(k_{1}^{2}+m^{2}\right) \rho_{\mu \nu}\left(k_{1}\right)=\left(k_{2}^{2}+m^{2}\right) \rho_{\mu \nu}\left(k_{2}\right)=\left(k_{2}^{2}-8+m^{2}\right) \phi\left(k_{2}\right)=0$ then we take $t$ near one of the on-shell values, and at the end, consider the limit $m^{2} \rightarrow 0$. All three of the matrix elements $T_{\rho \phi}^{(2)}, T_{\phi 2}^{(2)}, T_{\rho 2}^{(2)}$ can be treated thus, and below we list our results for these when $t$ is near mass shell for the massless and tachyon modes. In all our subsequent discussion we let $k=k_{1}+k_{2}$.

$$
\begin{aligned}
\left(T_{\rho \phi}^{(2)}\right)_{t-0}= & -\frac{(-i)^{3}}{2} \int \frac{\mathrm{d}^{D} k_{2}}{(2 \pi)^{D}} \frac{\mathrm{d}^{D} k_{1}}{(2 \pi)^{D}}(2 \pi)^{D} \delta^{D}\left(p^{\prime}-k_{1}-k_{2}-p\right) \rho_{\mu^{\prime} \nu^{\prime}}\left(k_{1}\right) \phi\left(k_{2}\right) \\
& \times\left(\eta_{\mu}^{\mu^{\prime}}-\frac{1}{2} k_{2}^{\mu^{\prime}} \frac{\left(k_{2}-k_{1}\right)_{\mu}}{4}\right)\left(\eta_{\nu}^{\nu^{\prime}}-\frac{1}{2} k_{2}^{\nu^{\prime}} \frac{\left(k_{2}-k_{1}\right)_{\nu}}{4}\right) Y_{\text {massless }}^{\mu \nu, \lambda} \\
\left(T_{\rho \phi}^{(2)}\right)_{t \sim-8}= & (-i)^{3} \int \frac{\mathrm{d}^{D} k_{1}}{(2 \pi)^{D}} \frac{\mathrm{d}^{D} k_{2}}{(2 \pi)^{D}}(2 \pi)^{D} \delta^{D}\left(p^{\prime}-k_{1}-k_{2}-p\right) \rho_{\mu \nu}\left(k_{1}\right) \phi\left(k_{2}\right) \\
& \times\left(-\frac{1}{2}\right) \frac{k_{2}^{\mu}}{2} \frac{k_{2}^{\nu}}{2} Y_{\text {tach }}^{\lambda}, \\
\left(T_{\rho 2}^{(2)}\right)_{t \sim 0}= & -(-i)^{3} \frac{1}{4} \int \frac{\mathrm{d}^{D} k_{1}}{(2 \pi)^{D}} \frac{\mathrm{d}^{D} k_{2}}{(2 \pi)^{D}}(2 \pi)^{D} \delta^{D}\left(p^{\prime}-k_{1}-k_{2}-p\right) \\
& \times \rho_{\mu^{\prime} \nu^{\prime}}\left(k_{1}\right) \rho_{\alpha^{\prime} \beta^{\prime}}\left(k_{2}\right) t^{\mu^{\prime} \alpha^{\prime} \mu} t^{\nu^{\prime} \beta^{\prime} \nu}\left(Y_{\text {massless }}\right)_{\mu \nu} \lambda,
\end{aligned}
$$


with

$$
\begin{aligned}
& t^{\mu^{\prime} \alpha^{\prime} \mu}=\left\{\eta^{\alpha^{\prime} \mu} \frac{k^{\mu^{\prime}}}{2}-\eta^{\mu \mu^{\prime}} \frac{k^{\alpha^{\prime}}}{2}+\left(\eta^{\alpha^{\prime} \mu^{\prime}}-\frac{k^{\mu^{\prime}}}{2} \frac{k^{\alpha^{\prime}}}{2}\right)\left(\frac{k_{1}-k_{2}}{4}\right)^{\mu}\right\} \\
& \left(T_{\rho 2}^{(2)}\right)_{t--8}=-(-i)^{3} \frac{1}{4} \int \frac{\mathrm{d}^{D} k_{1}}{(2 \pi)^{D}} \frac{\mathrm{d}^{D} k_{2}}{(2 \pi)^{D}}(2 \pi)^{D} \delta^{D}\left(p^{\prime}-k_{1}-k_{2}-p\right) \\
& \times \rho_{\mu \nu}\left(k_{1}\right) \rho_{\alpha \beta}\left(k_{2}\right)\left(\eta^{\alpha \mu}-\frac{k^{\alpha}}{2} \frac{k^{\mu}}{2}\right)\left(\eta^{\beta \nu}-\frac{k^{\beta}}{2} \frac{k^{\nu}}{2}\right)\left(Y_{\mathrm{tach}}\right)^{\lambda} \\
& \left(T_{\phi 2}^{(2)}\right)_{t \sim 0}=(-i)^{3} \frac{1}{32} \int \frac{\mathrm{d}^{D} k_{1}}{(2 \pi)^{D}} \frac{\mathrm{d}^{D} k_{2}}{(2 \pi)^{D}}(2 \pi)^{D} \delta^{D}\left(p^{\prime}-k_{1}-k_{2}-p\right) \phi\left(k_{1}\right) \phi\left(k_{2}\right) \\
& \times\left(k_{1 \mu} k_{2 \nu}+k_{1 \nu} k_{2 \mu}\right)\left(Y_{\text {massless }}\right)^{\mu \nu, \lambda}, \\
& \left(T_{\phi 2}^{(2)}\right)_{t--8}=-(-i)^{3} \frac{1}{4} \int \frac{\mathrm{d}^{D} k_{1}}{(2 \pi)^{D}} \frac{\mathrm{d}^{D} k_{2}}{(2 \pi)^{D}}(2 \pi)^{D} \delta^{D}\left(p^{\prime}-k_{2}-k_{2}-p\right) \phi\left(k_{1}\right) \phi\left(k_{2}\right) \\
& \times\left(Y_{\text {tach }}\right)^{\lambda}
\end{aligned}
$$

Consider the $t \sim 0$ contribution to the three matrix elements. These have the same factorized form as $T_{\text {massless }}^{(1)}$ and in particular the tensor structure $Y_{\text {massless }}^{\mu r^{\prime} \lambda}$ that depends only on the external states is the same. These therefore contribute to the equation of motion for the massless modes and this contribution can be consistently identified using,

$$
T_{\text {massless }}^{(1)}+\left(T_{\rho \phi}^{(2)}\right)_{t \sim 0}+\left(T_{\phi 2}^{(2)}\right)_{t \sim 0}+\left(T_{\rho 2}^{(2)}\right)_{t \sim 0} \approx 0
$$

Similarly, the tachyon equation of motion to this order can be identified from,

$$
T_{\text {tach }}^{(1)}+\left(T_{\rho \phi}^{(2)}\right)_{t \sim-8}+\left(T_{\phi 2}^{(2)}\right)_{t--8}+\left(T_{\rho 2}^{(2)}\right)_{t--8} \approx 0
$$


In $x$-space, these equations of motion are (in the gauge $\partial^{\mu} \rho_{\mu v}=0$ )

$$
\begin{aligned}
\partial^{2} \rho_{\mu \nu}(x)=\frac{1}{2} \partial_{\mu} \phi \partial_{\nu} \phi & -\left\{\partial^{\lambda} \partial^{\sigma}\left(\rho_{\lambda \sigma} \rho_{\mu \nu}-\rho_{\mu \lambda} \rho_{\sigma \mu}\right)\right. \\
& +\frac{1}{2}\left(\eta^{\lambda \sigma}+\frac{\partial^{\lambda}}{2} \frac{\partial^{\sigma}}{2}\right) \partial^{\gamma}\left(\partial_{\nu} \rho_{\gamma \delta} \rho_{\mu \lambda}-\rho_{\gamma \sigma} \partial_{\nu} \rho_{\mu \lambda}\right) \\
& +\frac{1}{2}\left(\eta^{\lambda \sigma}+\frac{\partial^{\lambda}}{2} \frac{\partial^{\sigma}}{2}\right) \partial^{\gamma}\left(\partial_{\mu} \rho_{\sigma \gamma} \rho_{\lambda \nu}-\rho_{\gamma \sigma} \partial_{\mu} \rho_{\lambda \nu}\right) \\
& +\frac{1}{4}\left(\eta^{\lambda \sigma}+\frac{\partial^{\lambda}}{2} \frac{\partial^{\sigma}}{2}\right)\left(\eta^{\gamma \delta}+\frac{\partial^{\gamma}}{2} \frac{\partial^{\delta}}{2}\right)\left(\partial_{\mu} \partial_{\nu} \rho_{\sigma \delta} \rho_{\lambda \gamma}-\partial_{\mu} \rho_{\sigma \delta} \partial_{\nu} \rho \lambda_{\gamma}\right\} \\
& +4 \rho_{\mu \nu} \phi+\left(\rho_{\lambda \nu} \partial_{\mu} \partial^{\lambda} \phi-\partial_{\mu} \rho_{\lambda \nu} \partial^{\lambda} \phi\right)+\left(\rho_{\mu \lambda} \partial_{\nu} \partial^{\lambda} \phi-\partial_{\nu} \rho_{\mu \lambda} \partial^{\lambda} \phi\right) \\
& +\frac{1}{8}\left(\rho_{\lambda \sigma} \partial_{\mu} \partial_{\nu} \partial^{\lambda} \partial^{\sigma} \phi-\partial_{\mu} \rho_{\lambda \sigma} \partial_{\nu} \partial^{\lambda} \partial^{\sigma} \phi\right. \\
& \left.\quad-\partial_{\nu} \rho_{\lambda \sigma} \partial_{\mu} \partial^{\lambda} \partial^{\sigma} \phi+\partial_{\mu} \partial_{\nu} \rho_{\lambda \sigma} \partial^{\lambda} \partial^{\sigma} \phi\right) \\
\left(\partial^{2}+8\right) \phi(x)=2 \phi^{2}+ & +2\left(\eta^{\alpha \mu}+\frac{\partial^{\alpha}}{2} \frac{\partial^{\mu}}{2}\right)\left(\eta^{\beta \nu}+\frac{\partial^{\beta}}{2} \frac{\partial^{\nu}}{2}\right)\left(\rho_{\mu \nu} \rho_{\alpha \beta}\right)-\rho_{\mu \nu}(x) \partial^{\mu} \partial^{\nu} \phi .
\end{aligned}
$$

These equations of motion are consistent with each other and the various couplings, with the string tree amplitude calculations which are performed in the same gauge $\partial^{\mu} \rho_{\mu \nu}=0$. For example, the $\rho \rho$ term in the equation of motion for the massless modes is completely consistent with string tree three-point amplitudes with three external massless legs. Similar statements are true for the other couplings in these equations.

The equations of motion for the massive modes can be determined in an analogous manner by taking the corresponding $t$-channel pole in the matrix element $T^{(2)}$. For the first massive excitation, the $\rho \phi, \phi \phi$ and $\rho \rho$ contributions to its equations of motion can be determined by picking up the contributions from the $t \sim 8$ pole in the matrix elements $T_{\rho \phi}^{(2)}, T_{\phi 2}^{(2)}$ and $T_{\rho 2}^{(2)}$ respectively. The finite 
contributions upon taking the $t \sim 8$ pole in $T_{\rho \phi}^{(2)}, T_{\phi 2}^{(2)}$ and $T_{\rho 2}^{(2)}$ are listed below

$$
\begin{aligned}
& \left(T_{\rho \phi}^{(2)}\right)_{t-8}=(-i)^{3} \int \frac{\mathrm{d}^{D} k_{1}}{(2 \pi)^{D}} \frac{\mathrm{d}^{D} k_{2}}{(2 \pi)^{D}} \rho_{\mu^{\prime} \nu^{\prime}}\left(k_{1}\right) \phi\left(k_{2}\right)(2 \pi)^{D} \delta^{D}\left(p^{\prime}-k_{1}-k_{2}-p\right) \\
& \times\left(-\frac{1}{8}\right)\left[\eta_{\mu}^{\mu^{\prime}}\left(\frac{k_{2}-k_{1}}{2}\right)_{\alpha}+\eta_{\alpha}^{\mu^{\prime}}\left(\frac{k_{2}-k_{1}}{2}\right)_{\mu}\right. \\
& \left.-\frac{1}{2} \frac{k_{2}^{\mu^{\prime}}}{2}\left(\frac{k_{1 \alpha}}{2} \frac{k_{1 \mu}}{2}+\frac{k_{2 \alpha}}{2} \frac{k_{2 \mu}}{2}\right)\right] \\
& \times\left[\eta_{\nu}^{\mu^{\prime}}\left(\frac{k_{2}-k_{1}}{4}\right)_{\beta}+\eta_{\beta}^{\nu^{\prime}}\left(\frac{k_{2}-k_{1}}{4}\right)_{\nu}-\frac{1}{2} \frac{k_{2}^{\nu^{\prime}}}{2}\left(\frac{k_{1 \nu}}{2} \frac{k_{1 \beta}}{2}+\frac{k_{2 \nu}}{2} \frac{k_{2 \beta}}{2}\right)\right] \\
& \times Y_{\text {massive }}^{\mu \alpha \nu \beta, \lambda} \text {, } \\
& \left(T_{\rho 2}^{(2)}\right)_{t-8}=-(-i)^{3} \frac{1}{16} \int \frac{\mathrm{d}^{D} k_{1}}{(2 \pi)^{D}} \frac{\mathrm{d}^{D} k_{2}}{(2 \pi)^{D}}(2 \pi)^{D} \delta^{D}\left(p^{\prime}-k_{1}-k_{2}-p\right) \\
& \times \rho_{\mu^{\prime} \nu^{\prime}}\left(k_{1}\right) \rho_{\alpha^{\prime} \beta^{\prime}}\left(k_{2}\right) M_{\mu \alpha}^{\mu^{\prime} \alpha^{\prime}} M_{\nu \beta}^{\mu^{\prime} \beta^{\prime}}\left(Y_{\text {massive }}\right)^{\mu \alpha \nu \beta, \lambda} \\
& \left(T_{\phi 2}^{(2)}\right)_{t-8}=-(-i)^{3} \frac{1}{16} \int \frac{d^{D} k_{1}}{(2 \pi)^{D}} \frac{\mathrm{d}^{D} k_{2}}{(2 \pi)^{D}}(2 \pi)^{D} \delta^{D}\left(p^{\prime}-k_{1}-k_{2}-p\right) \\
& \times\left(\frac{k_{1}-k_{2}}{4}\right)_{\mu}\left(\frac{k_{1}-k_{2}}{4}\right)_{\alpha}\left(\frac{k_{1}-k_{2}}{4}\right)_{\nu}\left(\frac{k_{1}-k_{2}}{4}\right)_{\beta} \\
& \times \phi\left(k_{1}\right) \phi\left(k_{2}\right)\left(Y_{\text {massive }}\right)^{\mu \alpha \nu \beta, \lambda},
\end{aligned}
$$

with

$$
\begin{aligned}
M_{\mu \alpha}^{\mu^{\prime} \alpha^{\prime}}= & \left\{\left(\eta^{\mu^{\prime} \alpha^{\prime}}-\frac{k^{\mu^{\prime}}}{2} \frac{k^{\alpha^{\prime}}}{2}\right)\left(\frac{k_{1}-k_{2}}{4}\right)_{\mu}\left(\frac{k_{1}-k_{2}}{4}\right)_{\alpha}\right. \\
& +\eta_{\mu}^{\alpha^{\prime}} \frac{k^{\mu^{\prime}}}{2}\left(\frac{k_{1}-k_{2}}{4}\right)_{\alpha}+\eta_{\alpha}^{\alpha^{\prime}} \frac{k^{\mu^{\prime}}}{2}\left(\frac{k_{1}-k_{2}}{2}\right)_{\mu}-\eta_{\mu}^{\mu^{\prime}} \frac{k^{\alpha^{\prime}}}{2}\left(\frac{k_{1}-k_{2}}{4}\right)_{\alpha} \\
& \left.-\eta_{\alpha}^{\mu^{\prime}} \frac{k^{\alpha^{\prime}}}{2}\left(\frac{k_{1}-k_{2}}{4}\right)_{\mu}+\eta_{\mu}^{\alpha^{\prime}} \eta_{\alpha}^{\mu^{\prime}}+\eta_{\alpha}^{\alpha^{\prime}} \eta_{\mu}^{\mu^{\prime}}-\frac{1}{2} \frac{k^{\mu^{\prime}}}{2} \frac{k^{\alpha^{\prime}}}{2} \frac{k_{\mu}}{2} \frac{k_{\alpha}}{2}\right\} .
\end{aligned}
$$


These again have the same factorized form as $T_{\text {massive }}^{(1)}$ and in particular the tensor structure $Y_{\text {massive }}^{\mu \alpha \nu, \lambda}$ which depends only on the external states is the same. Thus, the $\rho \phi, \phi \phi$ and $\rho \rho$ contributions to the equations of motion for the first massive mode may be consistently obtained from

$$
T_{\text {massive }}^{(1)}+\left(T_{\rho \phi}^{(2)}\right)_{t \sim 8}+\left(T_{\phi 2}^{(2)}\right)_{t \sim 8}+\left(T_{\rho 2}^{(2)}\right)_{t \sim 8} \approx 0
$$

The various couplings are again consistent with the string tree point calculations for the three-point functions with an external massive mode and two massless modes, or two tachyon modes and an external massive mode, a massless mode and a tachyon mode.

Eqs. (3.52), (3.53) and (3.58) are the main results of this section. They give the equations of motion for the massless, tachyon and first massive mode of the string up to terms of second order in the massless-massless, tachyon-tachyon and massless-tachyon couplings. We have emphasized earlier that all of these are consistent with the string tree amplitude calculations and, as we discuss in detail below, also with each other, in the sense that the relative normalizations of the couplings in the massless and tachyon equations of motion are compatible. Further, they contain contributions all to orders in $\alpha^{\prime}$.

\section{Discussion}

In this paper we have studied the interrelations between conformal invariance and the unitarity of the $S$ matrix for strings in background fields. We first formulated the unitarity constraint which essentially states that physical states in the remote part, map into physical states after interaction. It was shown that this unitarity constraint is realized if the Virasoro algebra in the presence of background fields is closed. So, in this sense, conformal invariance guarantees the unitarity of the $S$ matrix. We next showed that enforcing the unitarity constraint in a string theory in the presence of background fields places constraints on these fields which are just the equations of motion for the different string modes. In this manner, not only the equations of motion for the massless modes, but also those of the tachyon and other massive modes, can be consistently obtained. We have explicitly obtained these equations of motion in a weak field expansion up to second order in weak fields. These are valid on-shell and contain contributions to all orders in $\alpha^{\prime}$. The equations of motion derived in this paper, however are not gauge covariant but in a particular gauge, $\partial^{\mu} \rho_{\mu \nu}=0$ for the massless modes. This is the same gauge used in string tree amplitude calculations. The equations of motion derived in this paper have couplings which are completely consistent with the appropriate string three-point amplitudes.

The question that naturally arises is whether the equations of motion can be obtained from an effective action. For purely the massless modes it is possible if an 
expansion in $\alpha^{\prime}$ is made. This is because the various couplings in the equations of motion obtained here are consistent with the string tree amplitude calculations and the effective action that reproduces the string three-point amplitude has been obtained up to terms of order $\alpha^{\prime}$ in ref. [6]. Here we would like to point out that since we work in a gauge fixed formalism as in ref. [7], the gauge invariant action that reproduces the equations of motion will contain contributions from all the massless modes, i.e. the dilaton the graviton and the antisymmetric tensor modes. As discussed in refs. [6] and [7], in order to do that, one has to project out the appropriate spin components using projection operators. Whether it is possible for the equations of motion for the tachyon and the massive excitations to be also obtained from a covariant effective action is unclear to us. Even for the massless modes, we do not know if a covariant effective action can be written down without making an $\alpha^{\prime}$ expansion. However, the equations of motion for the massless and the tachyon modes given in (3.52) and (3.53) are compatible with each other in the sense that the $\phi \phi$ contribution to the massless equation and the $\rho \phi$ contribution to the tachyon equation can be obtained with the correct coefficients from an effective lagrangian. The same is true for the $\rho \phi$ contribution to the massless equation and the $\rho \rho$ contribution to the tachyon equation of motion. For example, the contribution $\frac{1}{2} \partial_{\mu} \phi \partial_{\nu} \phi$ to the massless equation and the contribution $-\rho_{\mu \nu} \partial^{\mu} \partial^{\nu} \phi$ to the tachyon equation can be obtained from an effective action of the form

$$
\Delta L=\int \mathrm{d}^{D} x \frac{1}{2} \partial^{\mu} \phi \partial^{\nu} \phi \rho_{\mu \nu}(x)
$$

after using the gauge conditions and dropping total derivative terms. This, we feel is a very satisfactory feature of our approach and an important consistency check of the theory.

This work was supported in part by the US Department of Energy.

\section{Appendix A}

In this appendix we will give the proof of the fact that the matrix element $T$ vanishes if we assume the closure of the Virasoro algebra. We will use induction with respect to the number of weak fields.

For the proof, it is convenient to separate out $\langle A| L_{n}^{(0)}$ from $T$ and define the following state $\left|V_{N}\right\rangle$.

$$
T^{(N)}=\left\langle A\left|L_{n}^{(0)}\right| V_{N}\right\rangle
$$

where the superscript " $N$ " represents the number of weak fields. We always omit an 
overall $2 \pi \delta(0)$ factor from $T^{(N)}$. Explicitly,

$$
\begin{aligned}
\left|V_{N}\right\rangle= & \left(-i H^{(N)}\right)|B\rangle+\left(-i H^{(N-1)}\right) \frac{-i}{\Delta}\left(-i H^{(1)}\right)|B\rangle \\
& +\left(-i H^{(1)}\right) \frac{-i}{\Delta}\left(-i H^{(N-1)}\right)|B\rangle+\ldots \\
& +\underbrace{\left(-i H^{(1)}\right) \frac{-i}{\Delta}\left(-i H^{(1)}\right) \ldots \frac{-i}{\Delta}\left(-i H^{(1)}\right)|B\rangle}_{N \text { times }} .
\end{aligned}
$$

Notice that $\left|V_{N}\right\rangle$ can be constructed recursively

$$
\begin{aligned}
\left|V_{N}\right\rangle= & \left(-i H^{(N)}\right)|B\rangle \\
& +\sum_{l=1}^{N-1}\left(-i H^{(l)}\right) \frac{-i}{\Delta}\left|V_{N-l}\right\rangle
\end{aligned}
$$

Now we will show the following fact by induction

$$
L_{n}^{(0)}\left|V_{N}\right\rangle=(\Delta+n)\left\{\left(-i L_{n}^{(N)}\right)|B\rangle+\sum_{l=1}^{N-1}\left(-i L_{n}^{(l)}\right) \frac{-i}{\Delta}\left|V_{n-l}\right\rangle\right\}
$$

From this equation we can get $T^{(N)}=0$ since the state $\langle A|$ satisfies $\langle A|(\Delta+n)=0$.

It is easy to see that the above equation is true for $N=1$ and 2 . Then, in the following, we will show the validity of eq. (A.4) for the $(N+1)$ th order, assuming that it is correct up to the $N$ th order.

Let us apply $L_{n}^{(0)}$ to $\left|V_{N+1}\right\rangle$

$$
\begin{aligned}
|\Psi\rangle & \equiv L_{n}^{(0)}\left|V_{N+1}\right\rangle \\
& =L_{n}^{(0)}\left(i H^{(N+1)}\right)|B\rangle+L_{n}^{(0)} \sum_{l=1}^{N}\left(-i H^{(l)}\right) \frac{-i}{\Delta}\left|V_{N-l}\right\rangle \\
& \equiv\left|\Psi_{1}\right\rangle+\left|\Psi_{2}\right\rangle .
\end{aligned}
$$

In general, from the closure of the Virasoro algebra, the $m$ th order equation of the commutation return between $L_{n}$ and $H$ takes the following form:

$$
\sum_{k=0}^{m}\left[L_{n}^{(k)}, H^{(m-k)}\right]=n L_{n}^{(m)}
$$


or,

$$
L_{n}^{(0)} H^{(m)}=H^{(m)} L_{n}^{(0)}+(\Delta+n) L_{n}^{(m)}-L_{n}^{(m)} \Delta-\sum_{k=1}^{m-1}\left[L_{n}^{(k)} H^{(m-k)}\right]
$$

Using the above formula, we get for $\left|\Psi_{1}\right\rangle$

$$
\begin{aligned}
\left|\Psi_{1}\right\rangle & =L_{n}^{(0)}\left(-i H^{(N+1)}\right)|B\rangle \\
& =(-i)\left\{H^{(N+1)} L_{n}^{(0)}+(\Delta+n) L_{n}^{(N+1)}-L_{n}^{(N+1)} \Delta-\sum_{k=1}^{N}\left[L_{n}^{(k)} H^{(N+1-k)}\right]\right\}|B\rangle \\
& =(\Delta+n)\left(-i L_{n}^{(N+1)}\right)|B\rangle-(-i) \sum_{k=1}^{N}\left[L_{n}^{(k)}, H^{(N+1-k)}\right]|B\rangle .
\end{aligned}
$$

Here we have used the on-shell physical state condition for $|B\rangle$. For $\left|\Psi_{2}\right\rangle$ we have

$$
\begin{aligned}
\left|\Psi_{2}\right\rangle= & L_{n}^{(0)} \sum_{l=1}^{N}\left(-i H^{(l)}\right)\left(\frac{-i}{\Delta}\right)\left|V_{N+1-l}\right\rangle \\
= & (-i)^{2} \sum_{l=1}^{N}\left(H^{(l)} L_{n}^{(0)}+(\Delta+n) L_{n}^{(l)}-L_{n}^{(l)} \Delta-\sum_{k=1}^{l-1}\left[L_{n}^{(k)} H^{(l-k)}\right]\right) \frac{1}{\Delta}\left|V_{N+l}\right\rangle \\
= & (\Delta+n) \sum_{l=1}^{N}\left(-i L_{n}^{(l)}\right)\left(\frac{-i}{\Delta}\right)\left|V_{N+1-l}\right\rangle \\
& -\sum_{l=1}^{N} H^{(l)} L_{n}^{(0)} \frac{1}{\Delta}\left|V_{N+1-l}\right\rangle \\
& +\sum_{l=1}^{N} L_{n}^{(l)}\left|V_{N+1-l}\right\rangle+\sum_{l=1}^{N} \sum_{k=1}^{l-1}\left[L_{n}^{(k)}, H^{(l-k)}\right] \frac{1}{\Delta}\left|V_{N+1-l}\right\rangle .
\end{aligned}
$$

The second term can be modified as follows:

$$
\begin{aligned}
& -\sum_{l=1}^{N} H^{(l)} L_{n}^{(0)} \frac{1}{\Delta}\left|V_{N+1-l}\right\rangle \\
& \quad=-\sum_{l=1}^{N} H^{(l)} \frac{1}{\Delta+n} L_{n}^{(0)}\left|V_{N+1-l}\right\rangle \\
& \quad=-\sum_{l=1}^{N} H^{(l)}\left\{(-i) L_{n}^{(N+1-l)}|B\rangle+\sum_{k=1}^{N-l}\left(-i L_{n}^{(k)}\right)\left(\frac{-i}{\Delta}\right) \mid V_{n+1-l-k}\right)
\end{aligned}
$$


Here we have used the assumption (A.4) up to the $N$ th order. Finally, the third term of eq. (A.9) becomes, using eq. (A.3),

$$
\begin{aligned}
\sum_{l=1}^{N} L_{n}^{(l)}\left|V_{N+1-1}\right\rangle= & (-i) \sum_{l=1}^{N} L_{n}^{(l)} H^{(N+1-l)}|B\rangle \\
& +\sum_{l=1}^{N} \sum_{k=1}^{N-l} L_{n}^{(l)}\left(-i H^{(k)}\right) \frac{(-i)}{\Delta}\left|V_{N+1-l-k}\right\rangle
\end{aligned}
$$

Combining together $\left|\Psi_{2}\right\rangle$ can be rewritten as follows:

$$
\begin{aligned}
\left|\Psi_{2}\right\rangle= & (\Delta+n) \sum_{l=1}^{N}\left(-i L_{n}^{(l)}\right) \frac{-i}{\Delta}\left|V_{N+1-l}\right\rangle \\
& +(-i) \sum_{l=1}^{N}\left[L_{n}^{(l)} H^{(N+1-l)}\right]|B\rangle \\
& +\sum_{l=1}^{N} \sum_{k=1}^{N-l} H^{(l)} L_{n}^{(k)} \frac{1}{\Delta}\left|V_{N+1-l-k}\right\rangle \\
& -\sum_{l=1}^{N} \sum_{k=1}^{N-l} L_{n}^{(l)} H^{(k)} \frac{1}{\Delta}\left|V_{N+1-l-k}\right\rangle \\
& +\sum_{l-1}^{N} \sum_{k=1}^{l-1}\left[L_{n}^{(k)}, H^{(l-k)}\right] \frac{1}{\Delta}\left|V_{N+1-l}\right\rangle .
\end{aligned}
$$

It is easy to see, by a simple change of the order of summation, that the last three terms in the above expression cancel each other. Then, together with $\left|\Psi_{1}\right\rangle$ in eq. (A.8),

$$
\begin{aligned}
|\Psi\rangle & =L_{n}^{(0)}\left|V_{N+1}\right\rangle \\
& =\left|\Psi_{1}\right\rangle+\left|\Psi_{2}\right\rangle \\
& =(\Delta+n)\left\{-i L_{n}^{(N+1)}|B\rangle+\sum_{l=1}^{N}\left(-i L_{n}^{(l)}\right) \frac{-i}{\Delta}\left|V_{N+1-l}\right\rangle\right\} .
\end{aligned}
$$

This is the eq. (A.4) for the $(N+1)$ th order. 


\section{Appendix B}

In this appendix, we will prove the result stated in subsect. 3.2, i.e., the difference between the $\mathrm{T}$ and $\mathrm{T}^{*}$ products, cancels the term involving $H_{\text {massless }}^{(2)}$ in the interaction hamiltonian. Thus, if we use the $\mathrm{T}^{*}$ product, then such a term may be excluded from our considerations, in accordance with the Nambu-Mathews theorem. For a discussion of this theorem in field theories, see ref. [8], [9].

The hamiltonian formulation, which we are using in this paper, corresponds to using the T-product and the $S$-matrix is given in terms of the interaction hamiltonian by

$$
S=\mathrm{T} \exp \left(-i \int_{-\infty}^{\infty} H_{\mathrm{I}}(\tau) \mathrm{d} \tau\right)
$$

The interaction hamiltonian, $H_{\mathrm{I}}$, may be expanded in the number of weak fields as

$$
H_{\mathrm{I}}=H^{(1)}+H^{(2)}+\cdots .
$$

Here, we are omitting a subscript "massless" on the right-hand side and also the other terms in $H_{\mathrm{I}}$ which are not of interest for this discussion. $H^{(1)}$ and $H^{(2)}$ are given in eqs. (2.8), (2.11) and (2.15). To second order in weak fields, we have

$$
\begin{aligned}
S^{(2)}= & \frac{1}{2}(-i)^{2} \int \mathrm{d} \tau_{1} \int \mathrm{d} \tau_{2} \mathrm{~T}\left(H^{(1)}\left(\tau_{1}\right) H^{(1)}\left(\tau_{2}\right)\right) \\
& -i \int \mathrm{d} \tau_{1} H^{(2)}\left(\tau_{1}\right) .
\end{aligned}
$$

consider the first term: Introducing $\tau=i \ln r, Z=r \mathrm{e}^{i \sigma}$, this becomes

$$
\begin{aligned}
& \int \frac{\mathrm{d}^{D} k_{1}}{(2 \pi)^{D}} \int \frac{\mathrm{d}^{D} k_{2}}{(2 \pi)^{D}} \rho_{\mu \nu}\left(k_{1}\right) \rho_{\alpha \beta}\left(k_{2}\right) \frac{(-i)^{2}}{2} \cdot i^{2}\left(\frac{4}{16 \pi^{2}}\right) \int \frac{\mathrm{d}^{2} Z_{1}}{\left|Z_{1}\right|^{2}} \int \frac{\mathrm{d}^{2} Z_{2}}{\left|Z_{2}\right|^{2}} \\
& \times \mathrm{T}\left\{: \mathrm{e}^{i k_{1} X^{\left.\left(Z_{1}\right)\right)} P^{\mu}\left(Z_{1}\right) \tilde{P}^{\nu}\left(\bar{Z}_{1}\right):}\right. \\
& \left.\times: \mathrm{e}^{k_{2} X\left(Z_{2}\right)} P^{\alpha}\left(Z_{2}\right) \tilde{P}^{\beta}\left(\bar{Z}_{2}\right):\right\}
\end{aligned}
$$

The T-product here,is really an $r$-product, i.e., for two operators $O_{1}\left(Z_{1}\right), O_{2}\left(Z_{2}\right)$

$$
\begin{aligned}
T\left(O_{1}\left(Z_{1}\right) O_{2}\left(Z_{2}\right)\right) & =O_{1}\left(Z_{1}\right) O_{2}\left(Z_{2}\right),\left|Z_{1}\right|>\left|Z_{2}\right| \\
& =O_{2}\left(Z_{2}\right) O_{1}\left(Z_{1}\right),\left|Z_{2}\right|>\left|Z_{1}\right| .
\end{aligned}
$$


Our approach will be to write the T-product in \{\} in terms of a $\mathrm{T}^{*}$ product and some contact terms. For this purpose, consider first

$$
\begin{aligned}
\mathrm{T}\left(X^{\mu}\left(Z_{1}\right) X^{\nu}\left(Z_{2}\right)\right)= & \theta\left(\left|Z_{1}\right|-\left|Z_{2}\right|\right) X^{\mu}\left(Z_{1}\right) X^{\nu}\left(Z_{2}\right) \\
& +\theta\left(\left|Z_{2}\right|-\left|Z_{1}\right|\right) X^{\nu}\left(Z_{2}\right) X^{\mu}\left(Z_{1}\right)
\end{aligned}
$$

The operators, $P^{\mu}(Z)$ and $\tilde{P}^{\nu}(Z)$ are given by,

$$
\begin{aligned}
& P^{\mu}(Z)=\left(\frac{\partial}{\partial \tau}-\frac{\partial}{\partial \sigma}\right) X^{\mu}(Z)=-2 i Z \frac{\partial}{\partial Z} X^{\mu}(Z), \\
& \tilde{P}^{\nu}(\bar{Z})=\left(\frac{\partial}{\partial \tau}+\frac{\partial}{\partial \sigma}\right) X^{\mu}(Z)=-2 i \bar{Z} \frac{\partial}{\partial \bar{Z}} X^{\mu}(Z) .
\end{aligned}
$$

From this, it is straightforward to see that,

$$
\begin{aligned}
-2 i Z_{2} \frac{\partial}{\partial Z_{2}} \mathrm{~T}\left(X^{\mu}\left(Z_{1}\right) X^{\nu}\left(Z_{2}\right)\right) & =\mathrm{T}^{*}\left(X^{\mu}\left(Z_{1}\right) P^{\nu}\left(Z_{2}\right)\right) \\
& =\mathrm{T}\left(X^{\mu}\left(Z_{1}\right) P^{\nu}\left(Z_{2}\right)\right),
\end{aligned}
$$

Consider next,

$$
\begin{aligned}
\mathrm{T}\left(P^{\mu}\left(Z_{1}\right) X^{\nu}\left(Z_{2}\right)\right)= & \theta\left(\left|Z_{1}\right|-\left|Z_{2}\right|\right) P^{\mu}\left(Z_{1}\right) X^{\nu}\left(Z_{2}\right) \\
+ & \theta\left(\left|Z_{2}\right|-\left|Z_{1}\right|\right) X^{\nu}\left(Z_{2}\right) P^{\mu}\left(Z_{1}\right) \\
-2 i Z_{2} \frac{\partial}{\partial Z_{2}} \mathrm{~T}\left(P^{\mu}\left(Z_{1}\right) X^{\nu}\left(Z_{2}\right)\right)= & \mathrm{T}^{*}\left(P^{\mu}\left(Z_{1}\right) P^{\nu}\left(Z_{2}\right)\right) \\
= & \mathrm{T}\left(P^{\mu}\left(Z_{1}\right) P^{\nu}\left(Z_{2}\right)\right) \\
& +i\left|Z_{2}\right| \delta\left(\left|Z_{1}\right|-\left|Z_{2}\right|\right)\left[P^{\mu}\left(Z_{1}\right), X^{\nu}\left(Z_{2}\right)\right]
\end{aligned}
$$

using,

$$
\left[P^{\mu}\left(Z_{1}\right), X^{\nu}\left(Z_{2}\right)\right]_{\left|Z_{1}\right|=\left|Z_{2}\right|}=-i \pi \eta^{\mu \nu} \delta\left(\sigma_{1}-\delta_{2}\right)
$$

The above becomes:

$$
\mathrm{T}^{*}\left(p^{\mu}\left(Z_{1}\right) p^{\nu}\left(Z_{2}\right)\right)=\mathrm{T}\left(p^{\mu}\left(Z_{1}\right) p^{\nu}\left(Z_{2}\right)\right)+\pi \eta^{\mu \nu}\left|Z_{2}\right|^{2} \delta^{2}\left(Z_{1}-Z_{2}\right) .
$$


Similarly, it can be shown that,

$$
\begin{aligned}
\mathrm{T}^{*}\left(P^{\mu}\left(Z_{1}\right) \tilde{P}^{\nu}\left(\bar{Z}_{2}\right)\right)= & \mathrm{T}\left(P^{\mu}\left(Z_{1}\right) \tilde{P}^{\nu}\left(\bar{Z}_{2}\right)\right) \\
& +\pi \eta^{\mu \nu}\left|Z_{2}\right|^{2} \delta^{2}\left(Z_{1}-Z_{2}\right) \\
\mathrm{T}^{*}\left(\tilde{P}^{\mu}\left(\bar{Z}_{1}\right) \tilde{P}^{\nu}\left(\bar{Z}_{2}\right)\right)= & \mathrm{T}\left(\tilde{P}^{\mu}\left(\bar{Z}_{1}\right) \tilde{P}^{\nu}\left(\bar{Z}_{2}\right)\right) \\
& +\pi \eta^{\mu \nu}\left|Z_{2}\right|^{2} \delta^{2}\left(Z_{1}-Z_{2}\right) .
\end{aligned}
$$

The T-product in (B.4) may now be expanded out using Wick's theorem and in each term like $\mathrm{T}\left(P^{\mu}\left(Z_{1}\right) P^{\alpha}\left(Z_{2}\right)\right)$ may be rewritten in terms of the $\mathrm{T}^{*}\left(P^{\mu}\left(Z_{1}\right)\right.$, $P^{\alpha}\left(Z_{2}\right)$ ) plus the contact term using (B.12)-(B.14). We may then recombine all terms and write the T-product in (B.4) in terms of a $\mathrm{T}^{*}$ product plus the extra pieces. Noticing that the extra pieces arise only from $P P, P \tilde{P}$, or $\tilde{P} \tilde{P}$ contractions, we get the result that:

$$
\begin{aligned}
& \mathrm{T}\left\{: \mathrm{e}^{i k_{1} X\left(Z_{1}\right)} P^{\mu}\left(Z_{1}\right) \tilde{P}^{\nu}\left(\bar{Z}_{1}\right):\right.\left.: \mathrm{e}^{i k_{2} X\left(Z_{2}\right)} P^{\alpha}\left(Z_{2}\right) P^{\beta}\left(\bar{Z}_{2}\right):\right\} \\
&=\mathrm{T}^{*}\left\{: \mathrm{e}^{k_{1} X\left(Z_{1}\right)} P^{\mu}\left(Z_{1}\right) \tilde{P}^{\nu}\left(\bar{Z}_{1}\right):: \mathrm{e}^{i k_{2} X\left(Z_{2}\right)} P^{\alpha}\left(Z_{2}\right) P^{\beta}\left(\bar{Z}_{2}\right):\right\} \\
&-\pi\left|Z_{2}\right|^{2} \delta^{2}\left(Z_{1}-Z_{2}\right)\left\{\left(\mathrm{T}^{*}: \mathrm{e}^{i k_{1} X\left(Z_{1}\right)} \tilde{P}^{\nu}\left(\bar{Z}_{1}\right):: \mathrm{e}^{i k_{2} X\left(Z_{2}\right)}:: \tilde{P}^{\beta}\left(\bar{Z}_{2}\right):\right) \eta^{\mu \alpha}\right. \\
& \\
&+\mathrm{T}^{*}\left(: \mathrm{e}^{i k_{1} X\left(Z_{1}\right)} P^{\mu}\left(Z_{1}\right):: \mathrm{e}^{i k_{2} X\left(Z_{2}\right)} P^{\alpha}\left(Z_{2}\right):\right) \eta^{\nu \beta} \\
&+\mathrm{T}^{*}\left(: \mathrm{e}^{i k_{1} X\left(Z_{1}\right)} P^{\mu}\left(Z_{1}\right):: \mathrm{e}^{i k_{2} X\left(Z_{2}\right)} \tilde{P}^{\beta}\left(\bar{Z}^{2}\right):\right) \eta^{\nu \alpha} \\
&\left.+\mathrm{T}^{*}\left(: \mathrm{e}^{i k_{1} X\left(Z_{1}\right)} \tilde{P}^{\nu}\left(\bar{Z}_{1}\right):: \mathrm{e}^{i k_{2} X\left(Z_{2}\right)} P^{\alpha}\left(Z_{2}\right):\right) \eta^{\mu \beta}\right\} \\
&+\left(\pi\left|Z_{2}\right|^{2} \delta^{2}\left(Z_{1}-Z_{2}\right)\right)^{2}\left(\eta^{\mu \alpha} \eta^{\nu \beta}+\eta^{\mu \beta} \eta^{\nu \alpha}\right) \mathrm{T}^{*}\left(: \mathrm{e}^{i k_{1} X\left(Z_{1}\right)}:: \mathrm{e}^{i k_{2} X\left(Z_{2}\right)}:\right) .
\end{aligned}
$$

Substituting this into (B.4), the first term in (B.3) may be written as

$$
\begin{aligned}
& \frac{1}{2}(-i)^{2} \int \mathrm{d} \tau_{1}, \mathrm{~d} \tau_{2} \mathrm{~T}^{*}\left(H^{(1)}\left(\tau_{1}\right) H^{(1)}\left(\tau_{2}\right)\right)+\frac{i}{2} \int \mathrm{d} \sigma_{1} \int \frac{\mathrm{d} \tau_{1}}{4 \pi} \\
& \quad \times\left\{\left(: \rho_{\mu \nu}(X) \tilde{P}^{\nu}\left(\bar{Z}_{1}\right):: \rho_{\alpha \beta}(X) \tilde{P}^{\beta}\left(\bar{Z}_{1}\right):\right) \eta^{\mu \alpha}\right. \\
& \quad+\left(: \rho_{\mu \nu}(X) P^{\mu}\left(Z_{1}\right):: \rho_{\alpha \beta}(X) P^{\alpha}\left(Z_{1}\right):\right) \eta^{\nu \beta} \\
& \quad+\left(: \rho_{\mu \nu}(X) P^{\mu}\left(Z_{1}\right):: \rho_{\alpha \beta}(X) \tilde{P}^{\beta}\left(\bar{Z}_{1}\right):\right) \eta^{\nu \alpha} \\
& \left.\quad+\left(: \rho_{\mu \nu}(X) \tilde{P}^{\nu}\left(\bar{Z}_{1}\right):: \rho_{\alpha \beta}(X) P^{\alpha}\left(Z_{1}\right):\right) \eta^{\mu \beta}\right\} \\
& \quad+\frac{1}{8} \delta^{2}(0)\left(\eta^{\mu \alpha} \eta^{\nu \beta}+\eta^{\mu \beta} \eta^{\nu \alpha}\right) \int: \rho_{\mu \nu}(X):: \rho_{\alpha \beta}(X): \mathrm{d}^{2} Z_{1} .
\end{aligned}
$$


Consider now the second term in (B.3). In sect. 2, an expression for $H^{(2)}$ was given, however, no ordering prescription was specified. We will now choose the ordering for $H^{(2)}$ such that the Nambu-Mathews theorem is valid. The expression for $H^{(2)}$ given in sect. 2 is:

$$
H^{(2)}=2 \cdot \frac{1}{4 \pi} \int_{0}^{2 \pi} \mathrm{d} \sigma J_{\mu} J^{\mu},
$$

where, $J_{\mu}=\frac{1}{2}\left(P^{\lambda} \rho_{\lambda \mu}(X)+\rho_{\mu \lambda}(X) \tilde{P}^{\lambda}\right)$ so that $J_{\mu} J^{\mu}$ can be written as:

$$
\begin{aligned}
& \frac{1}{4}\left(\left(\rho_{\mu \nu}(X) P^{\nu} \rho_{\alpha \beta}(X) P^{\alpha}\right) \eta^{\nu \beta}+\left(\rho_{\mu \nu}(X) \tilde{P}^{\nu} \rho_{\alpha \beta}(X) \tilde{P}^{\beta}\right) \eta^{\mu \alpha}\right. \\
& \left.\quad+\left(\rho_{\mu \nu}(X) P^{\mu} \rho_{\alpha \beta}(X) \tilde{P}^{\beta}\right) \eta^{\nu \alpha}+\left(\rho_{\mu \nu}(X) \tilde{P}^{\nu} \rho_{\alpha \beta}(X) P^{\alpha}\right) \eta^{\mu \beta}\right)
\end{aligned}
$$

Thus, if we define $H^{(2)}$ by the ordering prescription

$$
\begin{aligned}
& H^{(2)}=2 \cdot \frac{1}{4 \pi} \int_{0}^{2 \pi} \mathrm{d} \sigma\{(\left.: \rho_{\mu \nu}(X) \tilde{P}^{\nu}:: \rho_{\alpha \beta}(X) \tilde{P}^{\beta}\right) \eta^{\mu \alpha} \\
&+\left(: \rho_{\mu \nu}(X) P^{\mu}:: \rho_{\alpha \beta}(X) P^{\alpha}:\right) \eta^{\nu \beta}+\left(: \rho_{\mu \nu}(X) P^{\mu}:: \rho_{\alpha \beta} \tilde{P}^{\beta}:\right) \eta^{\nu \alpha} \\
&\left.+\left(: \rho_{\mu \nu}(X) \tilde{P}^{\nu}:: \rho_{\alpha \beta}(X) P^{\alpha}:\right) \eta^{\mu \beta}\right\} \\
&=2 \cdot \frac{1}{4 \pi} \int_{0}^{2 \pi} \mathrm{d} \sigma: J^{\mu}:: J^{\mu}:
\end{aligned}
$$

then the second term in (B.16) exactly cancels the second term in (B.3). There remains the third term in (B.16) which is proportional to $\delta^{2}(0)$. In order for the Nambu-Mathews theorem to be valid, we have to choose a regularization prescription such that $\delta^{2}(0)=0$. That such a condition is necessary for the validity of the Nambu-Mathews theorem was pointed out in ref. [9].

In conclusion, if we choose the ordering prescription for $H^{(2)}$ as in (B.18) and use a proper regularization procedure, then we have shown that we may use the $T^{*}$ product to evaluate the term $\frac{1}{2}(-i)^{2} \int \mathrm{d} \tau_{1} \mathrm{~d} \tau_{2} \mathrm{~T}^{*}\left(H^{(1)}\left(\tau_{1}\right) H^{(1)}\left(\tau_{2}\right)\right)$ and drop the term that depends on $H^{(2)}$.

\section{Appendix C}

In this appendix we list some integral formulae which are useful in the evaluation of the matrix elements encountered in sect. 3. All the integrals needed can be 
obtained from the compact formula given below.

$$
\begin{aligned}
& \int \mathrm{d}^{2} Z|Z|^{\alpha}|1-Z|^{\beta} \\
& \times\left[\begin{array}{ccc}
1 & Z & Z^{-1} \\
\frac{1}{1-Z} & \frac{Z}{1-Z} & \frac{Z^{-1}}{1-Z} \\
\frac{Z}{1-Z} & \frac{Z^{2}}{1-Z} & \frac{1}{1-Z} \\
\frac{Z}{(1-Z)^{2}} & \frac{Z^{2}}{(1-Z)^{2}} & \frac{1}{(1-Z)^{2}}
\end{array}\right] \\
& \otimes\left[\begin{array}{ccc}
1 & \bar{Z} & \bar{Z}-1 \\
\frac{1}{1-\bar{Z}} & \frac{\bar{Z}}{1-\bar{Z}} & \frac{\bar{Z}^{-1}}{1-\bar{Z}} \\
\frac{\bar{Z}}{1-\bar{Z}} & \frac{\bar{Z}^{2}}{1-\bar{Z}} & \frac{1}{1-\bar{Z}} \\
\frac{\bar{Z}}{(1-\bar{Z})^{2}} & \frac{\bar{Z}^{2}}{\left(1-\bar{Z}^{2}\right.} & \frac{1}{(1-\bar{Z})^{2}}
\end{array}\right] \\
& =\left[\begin{array}{ccc}
1 & \frac{\alpha+2}{\alpha+\beta+4} & \frac{\alpha+\beta+2}{\alpha} \\
\frac{\alpha+\beta+2}{\beta} & \frac{\alpha+2}{\beta} & \frac{(\alpha+\beta)(\alpha+\beta+2)}{\alpha \beta} \\
\frac{\alpha+2}{\beta} & \frac{(\alpha+2)(\alpha+4)}{\beta(\alpha+\beta+4)} & \frac{\alpha+\beta+2}{\beta} \\
\frac{(\alpha+2)(\alpha+\beta+2)}{\beta(\beta-2)} & \frac{(\alpha+2)(\alpha+4)}{\beta(\beta-2)} & \frac{(\alpha+\beta)(\alpha+\beta+2)}{\beta(\beta+2)}
\end{array}\right] \\
& \otimes\left[\begin{array}{ccc}
1 & \frac{\alpha+2}{\alpha+\beta+4} & \frac{\alpha+\beta+2}{\alpha} \\
\frac{\alpha+\beta+2}{\beta} & \frac{\alpha+2}{\beta} & \frac{(\alpha+\beta)(\alpha+\beta+2)}{\alpha \beta} \\
\frac{\alpha+2}{\beta} & \frac{(\alpha+2)(\alpha+4)}{\beta(\alpha+\beta+4)} & \frac{\alpha+\beta+2}{\beta} \\
\frac{(\alpha+2)(\alpha+\beta+2)}{\beta(\beta-2)} & \frac{(\alpha+2)(\alpha+4)}{\beta(\beta-2)} & \frac{(\alpha+\beta)(\alpha+\beta+2)}{\beta(\beta-2)}
\end{array}\right] \times I_{0}
\end{aligned}
$$


with,

$$
I_{0}=\pi \frac{\Gamma\left(-\frac{\alpha}{2}-\frac{\beta}{2}-1\right) \Gamma\left(\frac{\alpha}{2}+1\right) \Gamma\left(\frac{\beta}{2}+1\right)}{\Gamma\left(-\frac{\alpha}{2}\right) \Gamma\left(-\frac{\beta}{2}\right) \Gamma\left(\frac{\alpha}{2}+\frac{\beta}{2}+2\right)}
$$

As an example of the use of the above formula, consider

$$
\begin{gathered}
\int \mathrm{d}^{2} Z|Z|^{\alpha}|1-Z|^{\beta}\left(\frac{Z^{2}}{1-Z}\right)\left(\frac{\bar{Z}^{2}}{(1-\bar{Z})^{2}}\right) \\
=\frac{(\alpha+2)(\alpha+4)}{\beta(\alpha+\beta+4)} \times \frac{(\alpha+2)(\alpha+4)}{\beta(\beta-2)} I_{0},
\end{gathered}
$$

i.e. in order to find the value of a particular integral on the left-hand side one writes down the appropriate entries of the matrices on the right-hand side and multiples by $I_{0}$.

\section{References}

[1] E. Fradkin and V. Tseytlin, Phys. Lett. 158B (1985) 316; Nucl. Phys. B261 (1985) 1;

C. Lovelace, Phys. Lett. 135B (1984) 75; Nucl. Phys. B273 (1986) 413;

C.G. Callan, D. Freidan, E.J. Martinec and M.J. Perry, Nucl. Phys. B262 (1985) 593;

A. Sen, Phys. Rev. Lett. 55 (1985) 1846; Phys. Rev. D32 (1985) 2102;

T. Banks, D. Nemeschansky, A. Sen, Nucl. Phys. B277 (1986) 67;

S. Das and B. Sathiapalan, Phys. Rev. Lett. 56 (1986) 2664

[2] R. Akhoury and Y. Okada, Phys. Lett. 183B (1987) 65

[3] R. Akhoury and Y. Okada, Phys. Rev. D35 (1987) 1917

[4] C. Rebbi, Phys. Reports 12C (1974) 1;

J. Scherk, Rev. Mod. Phys. 47 (1975) 123

[5] J. Schwarz, Phys. Reports 89 (1982) 223

[6] R.I. Nepomechie, Phys. Rev. D32 (1985) 3201

[7] J. Scherk and J. Schwarz, Nucl. Phys. B81 (1974) 118

[8] P.T. Mathews, Phys. Rev. 76 (1949) 686;

Y. Nambu, Prog. Theor. Phys. 7 (1952) 131

[9] C. Bernard and A. Duncan, Phys. Rev. D11 (1975) 848 